\title{
Efficient compressive sampling of spatially sparse fields in wireless sensor networks
}

\author{
Stefania Colonnese, Roberto Cusani, Stefano Rinauro ${ }^{*}$, Giorgia Ruggiero and Gaetano Scarano
}

\begin{abstract}
Wireless sensor networks (WSNs), i.e., networks of autonomous, wireless sensing nodes spatially deployed over a geographical area, are often faced with acquisition of spatially sparse fields. In this paper, we present a novel bandwidth/energy-efficient compressive sampling (CS) scheme for the acquisition of spatially sparse fields in a WSN. The paper contribution is twofold. Firstly, we introduce a sparse, structured CS matrix and analytically show that it allows accurate reconstruction of bidimensional spatially sparse signals, such as those occurring in several surveillance application. Secondly, we analytically evaluate the energy and bandwidth consumption of our CS scheme when it is applied to data acquisition in a WSN. Numerical results demonstrate that our CS scheme achieves significant energy and bandwidth savings with respect to state-of-the-art approaches when employed for sensing a spatially sparse field by means of a WSN.
\end{abstract}

\section{Introduction}

Wireless sensor networks (WSN) consist of autonomous, cooperative sensors spatially deployed over a geographical area, with applications ranging from surveillance [1] and localization systems [2,3], to environmental monitoring for physical field sensing and disaster prevention $[4,5]$. WSN nodes typically acquire the data and communicate them to a node named fusion center (FC), which stores the sensors' readings or forwards them through wired network infrastructures for further processing.

The availability of energy-efficient algorithms for data gathering towards the FC is particularly relevant when the monitoring network is deployed on a large geographical area (e.g., a forest), where highly efficient routing protocols are required for a sustainable network lifetime. Energy efficiency is also relevant in those environments where battery recharge or substitution may be unworkable (e.g., in underwater networks) [6,7]. On the other hand, efficient exploitation of available bandwidth is an important concern in bandwidth-limited or interference-limited environments.

The unifying sampling and compression approach of compressive sampling (CS) [8] is definitely well suited

\footnotetext{
*Correspondence: stefano.rinauro@uniroma1.it

Dipartimento di Ingegneria dell'Informazione, Elettronica e delle

Telecomunicazioni (DIET), Università di Roma "La Sapienza", Via Eudossiana 18,
} Rome 00184, Italy to resource-limited WSNs' applications. CS-based techniques for energy-efficient WSN data gathering have been recently investigated, with particular reference to the trade-off between reconstruction accuracy and data gathering cost [9]. A highly efficient approach is provided by random sensing (RS), where at each observation time, only a randomly drawn subset of sensors acquires data and transmits them to the FC, typically using single-hop links. From a theoretical point of view, RS and CS share conditions and procedures for signal reconstruction. An energy- and bandwidth-efficient RS procedure appears in [10].

WSN monitoring applications are often faced with acquisition of spatially sparse signals. A typical example is that of temperature-monitoring sensor networks for anomalous event (e.g., fire) detection: in the early stages of abnormal system behavior, in which the event is hopefully detected, the field is characterized by one or more small spots at levels largely different from the surroundings, and it can be modeled as a spatially sparse signal. RS schemes, such as those analyzed in [10], poorly perform in sampling signals that are naturally sparse in the spatial domain since the actual number of measurements required to reconstruct the field increases and the RS bandwidth/energy efficiency deteriorates.

This paper successfully addresses the efficient compressive sampling of spatially sparse signals in a WSN. We introduce a novel CS scheme that can be realized in a

\section{Springer}

(c) 2013 Colonnese et al:; licensee Springer. This is an Open Access article distributed under the terms of the Creative Commons Attribution License (http://creativecommons.org/licenses/by/2.0), which permits unrestricted use, distribution, and reproduction in any medium, provided the original work is properly cited. 
WSN by distributed and parallel data gathering schemes, with restrained energy and bandwidth consumption for inter-sensor signaling. Specifically, the main contributions of this work are the following:

- We introduce a novel CS matrix and analytically demonstrate that it satisfies the CS conditions for sparse signal reconstruction.

- We analytically evaluate the performance, in terms of energy and bandwidth efficiency, of a WSN data gathering scheme based on the CS matrix presented herein.

- We show that, on spatially sparse fields, our CS scheme outperforms selected RS and CS state-of-the-art ones in terms of both energy and bandwidth efficiency.

The remainder of the paper is organized as follows: Section 2 describes the WSN system model, Section 3 briefs the CS basics, and Section 4 discusses related works on CS/RS acquisition in WSN. In Section 5 we illustrate our original CS scheme, and in Section 6 we apply it to a WSN and analyze the related energy consumption and bandwidth occupancy. Section 7 presents numerical results assessing that our CS scheme outperforms state-of-the-art approaches in terms of bandwidth/energy efficiency. Section 8 concludes the paper.

\section{System model and network scenario}

Let us consider a physical field represented by a bidimensional time-varying signal $s(x, y ; t),(x, y) \in \mathcal{R}^{2}$ monitored through a grid of $N=N_{1} \times N_{2}$ sensors deployed over a bidimensional covered area, $N_{1}$ and $N_{2}$ being the number of sensors distributed in the horizontal and vertical dimension, respectively. A selected sensor collects the data from the others and plays the role of FC. An example of such a WSN scenario is depicted in Figure 1: the FC is placed at the center of the network, and each sensor is placed at distance $d_{k}, k=1, \ldots, N-1$ from the FC.

The sensors periodically measure $s(x, y ; t)$ and transmit their readings to the FC for monitoring. The sensing period $\Delta t$ is selected to be almost equal to the coherence time $T_{c}$ of $s(x, y ; t)^{\mathrm{a}}$. At time $t_{k}=t_{0}+k \Delta t$, the sensor at the location $\left(n_{1} d, n_{2} d\right)$ acquires the noisy measurement:

$$
z\left[n_{1}, n_{2}\right]=s\left(n_{1} d, n_{2} d ; t_{k}\right)
$$

where, for the sake of simplicity, we have dropped the temporal variable and have set the horizontal and vertical inter-sensor distances equal to $d$. Then, the sensors implement a suitable dissemination protocol to forward the measured value to the FC. The FC collects all the data

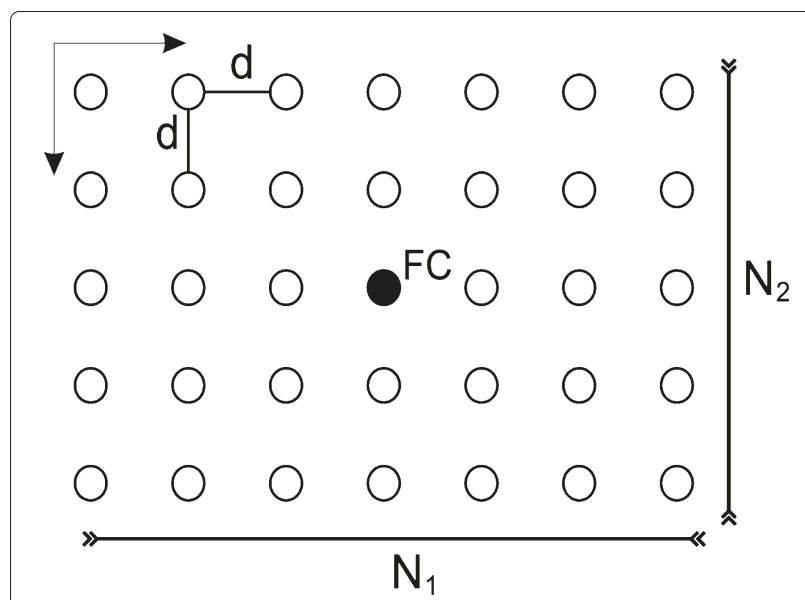

Figure 1 Sensor network scenario.

from the sensors so as to reconstruct a representation of the overall field $z\left[n_{1}, n_{2}\right]$.

Efficient data dissemination towards the FC is widely debated in the literature since energy efficiency affects the network lifetime, especially relevant in scenarios where the deployment of sensors is difficult or expensive, whereas bandwidth efficiency enables WSN monitoring in bandwidth-limited media, e.g., underwater, or in geographical areas where different sensor networks coexist. CS and RS paradigms provide a theoretical framework for highly efficient field monitoring, provided that the monitored data are sparse in a suitable domain. We briefly recall in Sections 3 and 4 the basics of CS and the related work on CS and RS application in WSN, respectively.

\section{An introduction to compressive sampling}

Let us compactly represent the bidimensional sequence $z\left[n_{1}, n_{2}\right]$ by the lexicographically ordered vector $\mathbf{z}$ :

$$
\mathbf{z}=\left[z[1,1] \cdots z\left[1, N_{2}\right] z[2,1] \cdots z\left[N_{1}, N_{2}\right]\right]^{\mathrm{T}} .
$$

The vector $\mathbf{z} \in \mathbb{R}^{N}$ is sparse if the number of its nonzero samples is restrained compared to its own dimension $N$; rigorously, $\mathbf{z}$ is said to be $K$-sparse if the number of non-zero components is $K$, either in the spatial or in a transformed domain (e.g., Fourier, wavelet, etc.). CS provides a framework for sensing and compression of a sparse signal.

According to the CS paradigm, compression of sparse signals is performed jointly with the acquisition. Specifically, $\mathbf{z}$ is represented by $M$ projections defined as follows:

$$
\mathbf{y}=\Phi \mathbf{z}
$$

$\Phi$ being a suitable measurement matrix of size $M \times N$.

A fundamental outcome of CS is that under suitable conditions on the sensing matrix $\Phi$, if $\mathbf{z}$ is $K$-sparse, it can 
be accurately recovered from the projections in y provided that $K<M<N$. Specifically, the sensing matrix $\Phi$ must satisfy the so-called restricted isometry property (RIP), i.e., given a constant $\delta$, for all $K$-sparse signals $\mathbf{z}$, it must stand:

$$
(1-\delta)\|\mathbf{z}\|_{2}^{2} \leq\|\Phi \mathbf{z}\|_{2}^{2} \leq(1+\delta)\|\mathbf{z}\|_{2}^{2}
$$

It is proven [11] that, for values of $\delta$ small enough, sparse signals can be perfectly recovered from compressive sensing measurements. In [12], the authors show that the RIP property is verified when the measurement energy $\|\Phi \mathbf{z}\|^{2}$ is more and more concentrated, in probability, around the value $\|\mathbf{z}\|_{2}^{2}$ as far as the number of measurements increases.

Reconstruction can be achieved either by solving the following optimization problem:

$$
\hat{\mathbf{z}}=\arg \min _{\mathbf{t}}\|\mathbf{t}\|_{1} \quad \text { s.t. } \quad \mathbf{y}=\Phi \mathbf{z}
$$

or by a greedy iterative pursuit of the support of $\mathbf{z}$; examples of this latter approach are provided by the orthogonal matching pursuit [13] and the compressive sampling matching (CoSaMP) algorithms [14].

In (2), we recognize that randomly sampling the components of $\mathbf{z}$ and collecting them in $\mathbf{y}$ is equivalent to realizing CS using a particular sensing matrix; therefore, many CS theory results apply to RS, too. Both RS and CS techniques have been considered for application in WSNs.

\section{Related works}

Efficient sensing and data gathering in WSNs by means of CS - and RS-based techniques have aroused lively interest in recent literature. In [15], a CS-based distributed communication architecture is exploited to minimize the latency for information retrieval under a favorable powerdistortion trade-off, whereas in [16], a CS-based sensing and data gathering procedure is analyzed for the case of network routing tree topologies. In [17], maximization of large-scale WSN lifetime is pursued by means of a fully distributed algorithm according to which each sensor autonomously performs classical or compressive sampling in order to reduce the number of transmitted packets.

In [9], the authors analyze a RS and multi-hop data gathering scheme. In this scheme, only randomly selected nodes measure the field and transmit their readings to the FC through specific multi-hop paths. While a sensor reading is routed towards the $\mathrm{FC}$, its value is combined with the ones sensed by the sensors in the path, so that each random projection provided to the $\mathrm{FC}$ is built by accumulating randomly weighted sensor readings along a network path. Energy efficiency is pursued if the number of nodes contributing to each projection is low.

In [18], a peculiar form of the CS sensing matrix is proven to exhibit good reconstruction properties while still being able to reduce the number of inter-sensor transmissions. The structure of the sensing matrix, originally designed for WSNs with chain topology, is viable of an extension to more complex scenarios, provided that suitably tree-structured routing paths are designed from the exterior of the network towards the FC.

Recently, in [10] a RS-based sensor network framework for underwater systems has been introduced. Differently from the works in $[9,16,17]$, a single-hop network is considered, where the sensors directly communicate with the FC. Every sampling interval $\Delta t$, each sensor senses the field and transmits it directly to the FC with probability $p$. The sensing probability $p$ is suitably chosen in order to let the FC acquire sufficiently many data for field reconstruction. The approach in [10] favorably merges the RS procedure with a random access protocol, thus obtaining a significant reduction in both the consumed energy and the occupied bandwidth. The main reasons why the above discussed methods achieve significant performances in efficient use of network resources are either in the fact that, during a sampling interval, only a subset of the sensors measures the field by means of RS or in the fact that CS acquisition is actually realized jointly with the routing procedure.

Recent studies $[16,18]$ suggest that in the case of spatially sparse signals, the energy/bandwidth of RS and CS efficiency deteriorates since reconstruction accuracy is guaranteed only when a large number of sensors contribute to the measurements. In [16], the authors remark how difficult it is to design a RS matrix suited to sparse signals and still allowing an efficient network routing. In [18], it is pointed out that, on spatially sparse signals, CS techniques still guarantee reconstruction accuracy but at an increased number of measurements (e.g., $M$ up to $50 \%$ of $N$ ), whereas for the same values of $M$ and $N$, RS only opportunistically achieves reconstruction.

The problem of efficient CS data routing from spatially localized signals is addressed in [19]. Therein, the authors of [19] propose to leverage the so-called transport cost, that is, the energy wasted to disseminate CS measurement towards the FC via multi-hop paths, by clustering the network nodes in non-overlapping sets, each one responsible for one or more CS measurements. In order to assure perfect reconstruction, the sensors' 
clusterization is selected depending on the sparsifying basis under which the field is sensed. The clusterization corresponds to a sparse structure in the sensing matrix, and it is the starting point to allow energy savings in the overall sensing procedure, realized by a centralized algorithm. The procedure is designed for spatially localized signals, which are sparse in a spatially localized basis (e.g., Daubechies, DCT), but it looses efficiency as the signals become more and more sparse in the spatial domain.

On the other hand, in several WSN applications, the sensed field indeed contains local fluctuations and abnormal readings, and it is well modeled as a spatially sparse signal. This motivated us to concern ourselves with the design of a sensing matrix suited to spatially sparse signals, as described in the following section.

\section{CS using Radon-like random projections}

In a nutshell, we aim at devising a sensing matrix that represents a spatially sparse field $\mathbf{z}$ in a domain such that dropping $N-M$ components does not prevent signal reconstruction. Recalling that the Radon transform has the dual properties of (1) compressing spatial domain straight lines into transform domain pulses [20] and (2) expanding spatial discrete pulses into as many non-zero values as the number of considered Radon projections [21], we recognize that the Radon transform provides a mean for redundant representation of sparse signals built by spatially isolated pulses. Thereby, we resort to a spatially sparse signal CS scheme inspired by the Radon projection computation.

To elaborate, let us consider the bidimensional sequence $z\left[n_{1}, n_{2}\right]$ of finite size $N_{1} \times N_{2}$, and let us present few examples of measurements computed in analogy to the Radon projections. First, let us consider the columnwise accumulation of a randomly weighted version of $z\left[n_{1}, n_{2}\right]$ :

$$
y^{(0)}[m]=\sum_{i=0}^{N_{2}-1} \varphi_{m}^{(0)}[i] z[i, m], m=0, \ldots, N_{1}-1
$$

where $\varphi_{m}^{(0)}[i], i=0, \ldots N_{1}-1, m=0, \ldots, N_{2}-1$ are independent and identically distributed (i.i.d.) zero mean Gaussian random variables of equal variance $\sigma_{\varphi}^{2}$, i.e., $\varphi_{m}^{(0)}[i] \sim \mathcal{N}\left(0, \sigma_{\varphi}^{2}\right)$; an example of the formation of the measurements $y^{(0)}[\mathrm{m}]$ is illustrated in Figure 2. The measurements $y^{(0)}[m]$ differ from a Radon projection in that each sample is randomly weighted. Besides, they differ from classical CS measurements in that each measure is obtained only from a subset (namely a column) of the values in $z\left[n_{1}, n_{2}\right]$ rather than from all the samples $z\left[n_{1}, n_{2}\right]$.

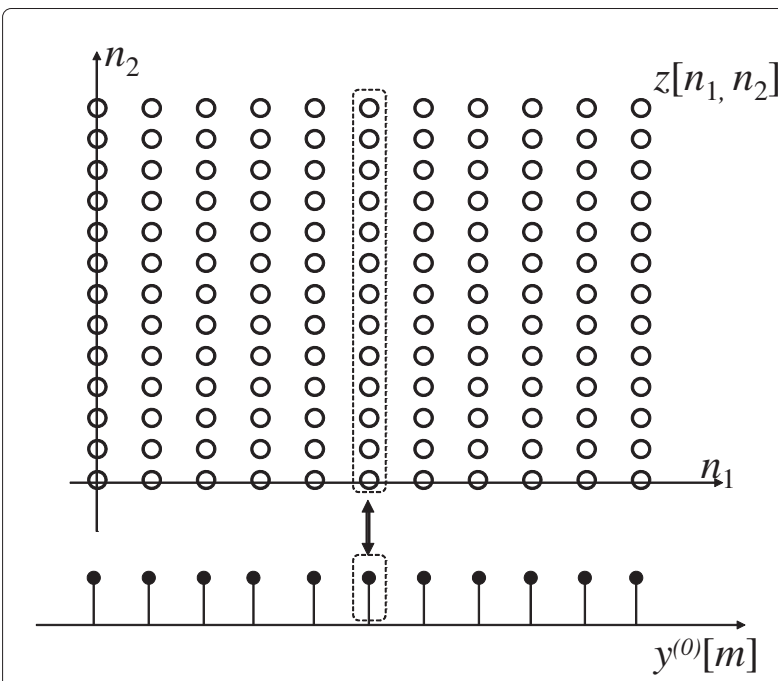

Figure 2 Bidimensional sequence $z\left[n_{1}, n_{2}\right]$ : column-wise accumulation for computation of the measurements $y^{(0)}[\mathrm{m}]$.

Definition of the row-wise random projections of $z\left[n_{1}, n_{2}\right]$ is straightforward, namely:

$$
y^{(\pi / 2)}[m]=\sum_{i=0}^{N_{1}-1} \varphi_{m}^{(\pi / 2)}[i] \cdot z[m, i], m=0, \ldots, N_{2}-1
$$

By analogy, we can define the diagonal-wise projection of $z\left[n_{1}, n_{2}\right]$ as well:

$$
y^{(\pi / 4)}[m]=\left\{\begin{array}{c}
\sum_{i=0}^{N_{1}-1-m} \varphi_{m}^{(\pi / 4)}[i] z[i+m, i] \\
m=0, \ldots, N_{1}-1 \\
\sum_{i=0}^{N_{2}-1-|m|} \varphi_{m}^{(\pi / 4)}[i] z[i, i+m] \\
m=-N_{2}+1, \ldots,-1 .
\end{array}\right.
$$

Expressions (4) to (6) represent randomly weighted accumulations of $z\left[n_{1}, n_{2}\right]$ over ridge paths. Let us now generalize the above expressions by regarding them as obtained by column-wise accumulation of a suitably rotated version of the sequence $z\left[n_{1}, n_{2}\right]$.

Let $z^{\left(\vartheta_{p}\right)}\left[n_{1}, n_{2}\right]$ be a $\vartheta_{p}$-radiant clockwise-rotated ${ }^{\mathrm{b}}$ version of the image $z\left[n_{1}, n_{2}\right]$. The size of $z^{\left(\vartheta_{p}\right)}\left[n_{1}, n_{2}\right]$ varies with $\vartheta_{p}$, and we denote as $K(p), p=0, \ldots P-1$ the number of columns of $z^{\left(\vartheta_{p}\right)}\left[n_{1}, n_{2}\right]$. We generalize definitions (4) to (6) by considering the collection of the random projections of $z^{\left(\vartheta_{p}\right)}\left[n_{1}, n_{2}\right]$ over a finite set of $P$ directions $\vartheta_{p}$ : 


$$
y^{\left(\vartheta_{p}\right)}[m]=\sum_{i} \varphi_{m}^{\left(\vartheta_{p}\right)}[i] \cdot z^{\left(\vartheta_{p}\right)}[i, m]
$$

for $m=0, \ldots K(p)-1, p=0, \ldots P-1$. Since the accumulation in (7) recalls the column-wise accumulations employed in the computation of the discrete Radon transform, we refer to the projections in (7) as Radon-like random projections.

Let us now collect the $P$ Radon-like random projections:

$$
\mathbf{y}^{\left(\vartheta_{p}\right)}=\left[y^{\left(\vartheta_{p}\right)}[0] \cdots y^{\left(\vartheta_{p}\right)}[K(p)-1]\right]^{\mathrm{T}}, p=0, \ldots P-1
$$

in a measurement vector $\mathbf{y}$ :

$$
\mathbf{y}=\left[\mathbf{y}^{\left(\vartheta_{0}\right)^{\mathrm{T}}} \cdots \mathbf{y}^{\left(\vartheta_{P-1}\right)^{\mathrm{T}}}\right]^{\mathrm{T}} .
$$

The measurements $\mathbf{y}$ are computed from $\mathbf{z}$ as

$$
\mathbf{y}=\Phi_{R} \mathbf{z}
$$

using the $\sum_{p} K(p) \times N_{1} N_{2}$ random sampling matrix $\Phi_{R}$ defined as

$$
\Phi_{R}=\left[\begin{array}{c}
\Phi_{R}^{\left(\vartheta_{0}\right)} \\
---- \\
-\overline{\Phi_{R}}\left(\bar{\vartheta}_{P-1}^{-}\right)
\end{array}\right]
$$

where $\Phi_{R}^{\left(\vartheta_{p}\right)}, p=0, \ldots P-1$ are the suitably defined sparse random matrix realizing the accumulation in (5) and (6). Specifically, the matrices $\Phi_{R}^{\left(\vartheta_{p}\right)}, p=0, \ldots P-1$ are built as follows. For each and every row, a few elements, corresponding to the coefficients of $\mathbf{x}$ that do not contribute to the measurement, are deterministically set to zero. The remaining non-zero elements are drawn from i.i.d. normal distribution. For the sake of clarity, we report the matrices $\Phi_{R}^{(0)}$ and $\Phi_{R}^{(\pi / 2)}$, corresponding to the horizontal and the vertical projection, in (10) and (9), respectively.
Let us remark that, even though the samples contributing to each y component are the same as those that would have contributed to a specific Radon projection of $\mathbf{z}$, due to the random weighting, the measurement vector $y$ is not - and it is not even easily related to - the Radon transform of $\mathbf{z}$.

In the following, we demonstrate that the conditions given by the CS theory for reconstructing $\mathbf{z}$ from $\mathbf{y}$ stand. Before turning to mathematics, let us observe that if the image $z\left[n_{1}, n_{2}\right]$ is built by sparse isolated pulses, each pattern contributes, apart from a suitable random weighting, to each one of the $P$ Radon-like random projections $y^{\left(\vartheta_{p}\right)}[m], \quad m=0, \ldots K(p)-1, \quad p=0, \ldots P-1$. Thereby, the set of $P$ Radon-like random projections is a $P$-redundant representation of the pulse. This motivated us to formally demonstrate that the Radon-like random projections $y^{\left(\vartheta_{p}\right)}[m], m=0, \ldots K(p)-1, p=0, \ldots P-1$ satisfy the conditions of a CS measurement set.

\subsection{Restricted isometry property of the Radon-like sampling matrix}

To formally state that the above introduced simplified sampling structure is feasible for accurate field reconstruction, we shall demonstrate that the random measurements $\mathbf{y}$ evaluated as in (8) are consistent CS measurements, i.e., they substantially preserve the information of the sampled sequence $z\left[n_{1}, n_{2}\right]$.

Formally, we need to prove that the sampling matrix $\Phi_{R}$ satisfies the condition known as restricted isometry property. Specifically, let us denote by $\mathcal{E}_{z} \stackrel{\text { def }}{=}\|\mathbf{z}\|^{2}$ the quadratic norm of the vector $\mathbf{z}$. If the matrix $\Phi_{R}$ satisfies

$$
(1-\delta) \mathcal{E}_{z} \leq\left\|\Phi_{R} \mathbf{z}\right\|^{2} \leq(1+\delta) \mathcal{E}_{z}
$$

with high probability, then any sparse sequence $\mathbf{z}$ can be perfectly recovered from CS measurements $\mathbf{y}=\Phi_{R} \mathbf{z}[11]$.

The RIP in (11) asserts that the measurement energy $\mathcal{E}_{y} \stackrel{\text { def }}{=}\left\|\Phi_{R} \mathbf{z}\right\|^{2}$ is strongly concentrated around the value $\mathcal{E}_{z}$. Preliminary results on the RIP property of a Radon-like CS matrix appear in [22]. In Appendix 1, we extend these

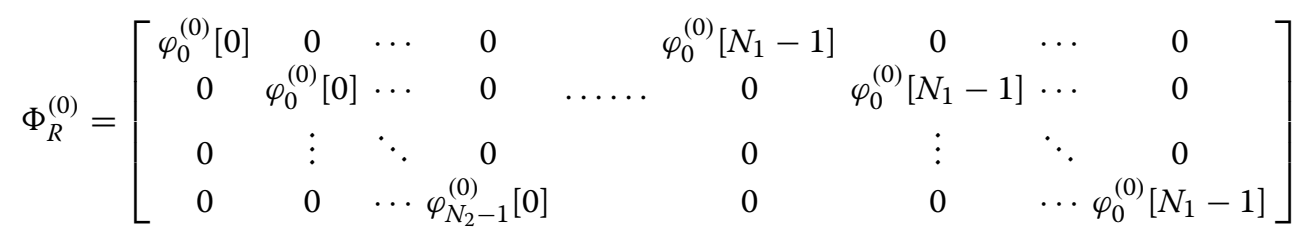

$$
\begin{aligned}
& \Phi_{R}^{(\pi / 2)}=\left[\begin{array}{ccccccccc}
\varphi_{0}^{(\pi / 2)}[0] & \varphi_{0}^{(\pi / 2)}[1] & \cdots & \varphi_{0}^{(\pi / 2)}\left[N_{2}-1\right] & & 0 & 0 & \cdots & 0 \\
0 & 0 & \cdots & 0 & \cdots \cdots & 0 & 0 & \cdots & 0 \\
0 & 0 & \ldots & 0 & & 0 & 0 & \cdots & 0 \\
0 & 0 & \ldots & 0 & \ldots \ldots & \varphi_{N_{1}-1}^{(\pi / 2)}[0] & \varphi_{N_{1}-1}^{(\pi / 2)}[1] \cdots & \varphi_{N_{1}-1}^{(\pi / 2)}\left[N_{2}-1\right]
\end{array}\right]
\end{aligned}
$$


results and, following the approach in [12], we show that the following property stands.

Property 1 Let us assume that the entries in the matrix $\Phi_{R}$ are either deterministically set to zero, or i.i.d. zero mean Gaussian random variables with equal variance $\sigma_{\varphi}^{2}=1 / P$. Then the following concentration inequality stands:

$$
\operatorname{Pr}\left\{\left|\mathcal{E}_{y}-\mathcal{E}_{z}\right| \geq \delta\right\} \leq \epsilon
$$

provided that $P \geq 2 K^{2} C_{2}^{2} \log (2 / \epsilon) / \delta^{2}, C_{2}$ being a suitable constant. From Property 1 , the RIP property in (11) immediately follows.

\subsection{Further remarks}

The sampling matrix $\Phi_{R}$ differs from the full sampling matrices usually adopted in CS since it is sparse. In the following, we show how the sparsity of the Radon-like sensing matrix $\Phi_{R}$ can be leveraged on to significantly simplify the CS measurement computation in a WSN so as to reduce the consumed energy and employed bandwidth.

The definition of $y^{\left(\vartheta_{p}\right)}[m]$ in (7) given above is consistent and useful from an analytical point of view. When turning to the evaluation of $y^{\left(\vartheta_{p}\right)}[m]$ in a WSN, the evaluation of $z^{\left(\vartheta_{p}\right)}\left[n_{1}, n_{2}\right]$ is not accomplished, and the measurement computation is realized throughout the data gathering stage.

In [23], the authors demonstrate the RIP for a different kind of sensing matrix, that is, block diagonal matrices. With respect to the approach in [23], the proof in Appendix 1 is carried out asymptotically, that is, for $M$ large enough to approximate $\mathcal{E}_{z}$ as the outcome of a Gaussian random variables, and it leverages the hypothesis of a spatially sparse signal, i.e., a signal that is sparse in the canonical basis.

Finally, the concentration inequality (12) guarantees that the sequence $\mathbf{z}$ can be recovered from the measurements $\mathbf{y}$ with high probability, as far as the number $P$ of considered projections increases. Since CS convergence is assured only in probability, the CS measurement experiment could be repeated. In a WSN, data are periodically sensed and routed to the FC, and a small probability of mis-reconstruction can be tolerated since it can be recovered in the subsequent sampling interval. Furthermore, integration of independently drawn measurements acquired in a WSN during different temporal intervals can be performed at the FC. This interesting research issue is deferred to further studies.

\section{Radon-like CS in a WSN}

In a WSN application scenario, the $P$ Radon-like projections correspond to randomly weighted sums of the values sensed by different subsets of WSN sensors. The sums can be evaluated using different techniques.
Following the approach in [24], the sensors within a subset can synchronously transmit their weighted sensed values, and the sum can be realized at the FC by on-air analogical superimposition of received signals. This protocol requires strict control of the power received by the FC from each sensor. Precisely, each sensor node needs to estimate the channel seen towards the FC in order to pre-compensate the transmitted value according to the channel attenuation. Thereby, although feasible in principle, this approach requires a sophisticated processing and tight power control by the sensor nodes.

According to a data gathering paradigm, the projections are computed within the network by a subset of sensors while they are forwarding their sensed values to the FC. The sums in (7) can then be realized by routing and accumulating values of $z\left[n_{1}, n_{2}\right]$ over suitably tilted paths in the network grid discrete support.

Here we refer to such a data gathering approach, and we infer some consequences from the peculiar sparse structure of the matrix $\Phi_{R}$ on the computation procedure. Firstly, we observe that in every row, the non-zero coefficients of the matrix $\Phi_{R}$ are arranged so as to obtain $y^{\left(\vartheta_{p}\right)}[m]$ as the sum of the values of a column of the rotated image $\mathbf{z}^{\left(\vartheta_{p}\right)}$. When collecting the measurement in a WSN, each projection $y^{\left(\vartheta_{p}\right)}[m]$ can then be computed by accumulating measurements throughout a specific, suitably tilted, grid path. Secondly, we observe that in every column of the matrix $\Phi_{R}$, there are only $P$ non-zero values. Hence, each value $z[i, j]$ shall contribute only to $P$ out of $M$ projections $y^{\left(\vartheta_{p}\right)}[m]$. When realizing the Radon-like CS in the sensor network grid, each sensor shall transmit its value $P$ times.

Based on these premises, we recognize that the sparse structure of the matrix $\Phi_{R}$ results into two main features of Radon-like projection computation in a WSN:

- The computation of each projection $y^{\left(\vartheta_{p}\right)}[m]$ is performed in a distributed way within the WSN, and it requires signaling among grid sensors which are adjacent along a WSN path.

- The accumulation along different paths can then be realized in parallel, provided that the distance between contemporaneously signaling nodes is kept large enough.

The data gathering scheme shown in Figure 3 is a case in point. A square network grid is employed to flood the data towards the FC. While forwarding the data along the linear paths (in red in Figure 3), the node partially accumulates them and therefore cooperates to incremental computation of the Radon-like CS measurements. To alleviate the load of the nodes in the FC nearby, the network can be divided into four quadrants that sequentially contribute to the data collection. As we will show in the 


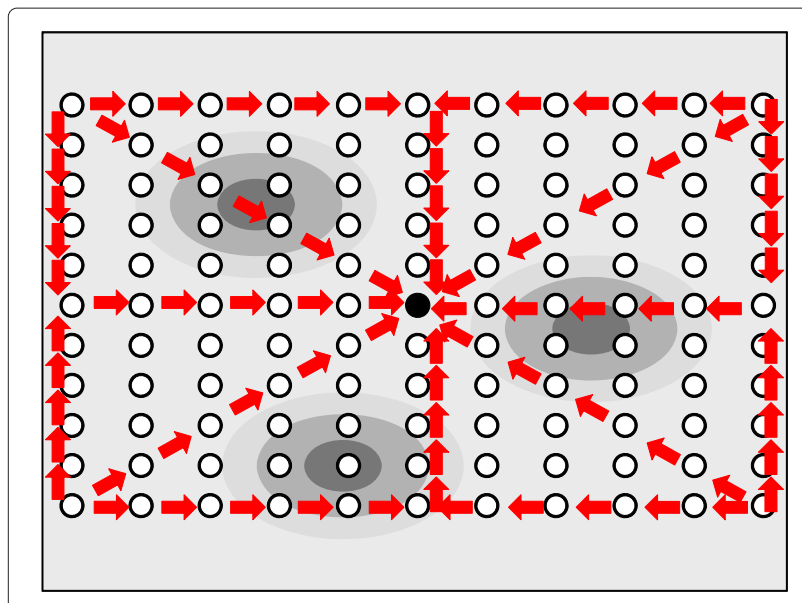

Figure 3 Example of quadrant-based data gathering geometry for Radon-like CS measurements computation in a WSN.

following, this quadrant-based approach may be used to derive a specific data gathering procedure.

We now turn to quantifying the energy consumption and bandwidth occupancy required for realizing the Radon-like CS by means of a data gathering procedure in a WSN. In Section 6.1, we evaluate the energy consumption and bandwidth occupancy of the Radon-like CS scheme in a WSN. Next, we compare these results with selected state-of-the-art schemes, namely those of the random sensing approaches described in Section 1, detailed in Section 6.2.

\subsection{Radon-like CS efficiency}

We now evaluate the allocated bandwidth and consumed energy for entirely collecting the measurements in a time $T_{c}$ in the WSN scenario in Section 2. The actual bandwidth occupation and energy consumption depend not only on the WSN structure but also on the adopted data gathering procedure. Without loss of generality, we refer to the suboptimal data gathering algorithm sketched out in Appendix 2, here briefly summarized for the reader's convenience.

According to the algorithm in Appendix 2, the sensors transmit their readings to the FC by data gathering through suitable multi-hop paths. Figures 4 and 5 illustrate, as an example, the multi-hop paths selected for the computation of the projections $y^{(\pi / 2)}[m], y^{(\pi / 4)}[m]$ within a network quadrant.

A deterministic (collision-free) time division multiple access (TDMA) is adopted. Signaling takes place between adjacent nodes only. Parallel transmission of nodes sufficiently apart is considered. In Appendix 2, we evaluate two parameters that directly affect the energy and bandwidth consumption of the data gathering algorithm, namely the total number of single-hop transmissions $\left(N_{\mathrm{TX}}\right)$ and the

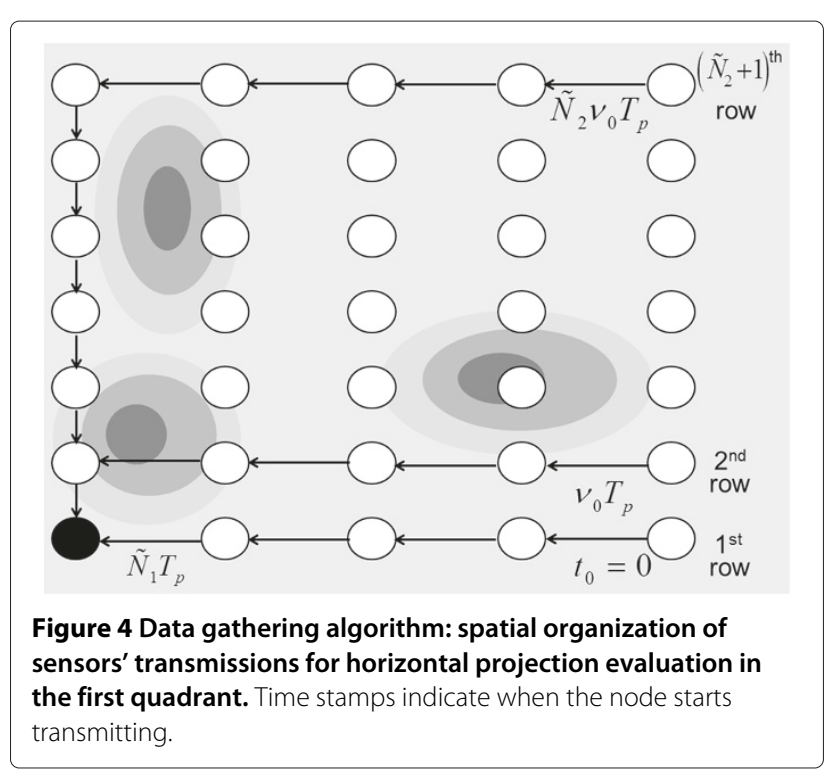

total number of time slots $\left(N_{\mathrm{TS}}\right)$ needed to collect all the sensors' readings to the FC.

The total number of node transmission, $N_{\mathrm{TX}}$, for the algorithm under concern comes out to vary linearly with the network size:

$$
N_{\mathrm{TX}} \approx \gamma_{P} \cdot N,
$$

$\gamma_{P}$ being a scalar factor depending on the adopted $P$ projections. The guidelines for calculating $\gamma_{P}$ are given in Appendix 2 where we also evaluate the value of $\gamma_{P}$ for different sets of directions $\vartheta_{p}$.

Based on the same data gathering algorithm, we have evaluated the number of time slots needed to collect all

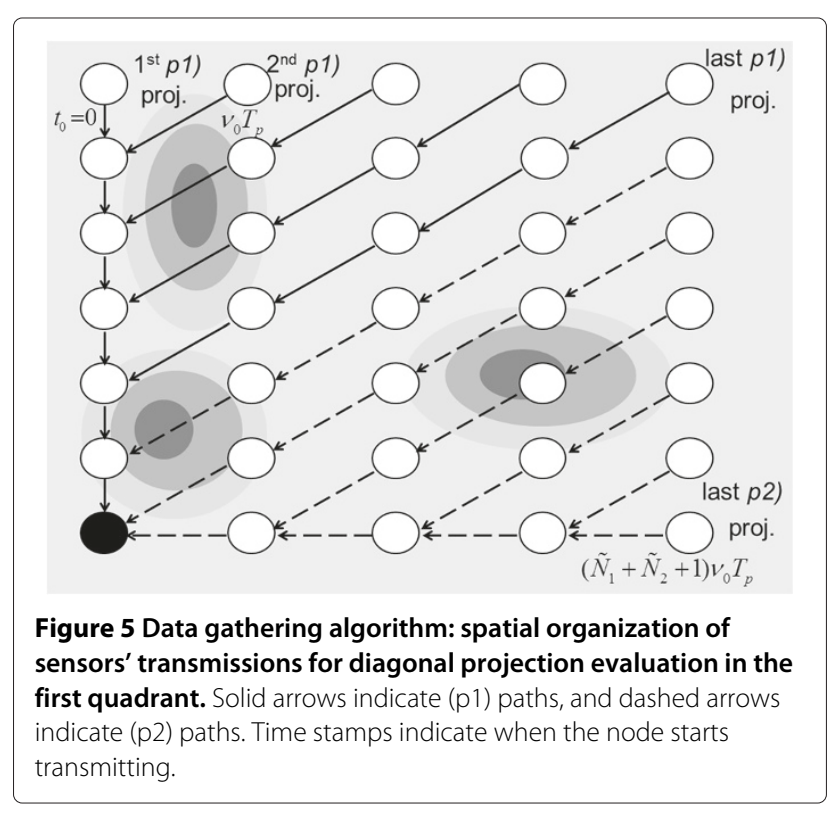


the sensors' readings to the FC. The number of time slots turns out to vary just with the square root of the network size, that is,

$$
N_{\mathrm{TS}} \approx \delta_{P} \cdot \sqrt{N}
$$

$\delta_{P}$ being a factor depending on the considered projection directions $\vartheta_{p}$. Appendix 2 reports the guidelines for the evaluation of the parameter $\delta_{P}$ for different sets of directions $\vartheta_{p}$.

We observe that, as a consequence of the sparsity of the Radon-like sensing matrix, (1) the number of transmission varies only linearly with the network size $N$, and (2) the algorithm being parallelized, the number of time slots varies linearly with the square root of the network size $N$. With these results, we are able to evaluate the allocated bandwidth and consumed energy for entirely collecting the measurements in a time $T_{c}$ in the WSN scenario described in Section 2.

Projection evaluation according to the data gathering scheme detailed in Appendix 2 accounts for a series of transmissions among neighboring nodes. The energy spent for a single-hop transmission is given by $E_{\mathrm{SH}}=$ $G d_{\mathrm{SH}}^{2} T_{p}^{(\mathrm{RL})}$, with $d_{\mathrm{SH}}=d$ or $\sqrt{2} d$ being the distance for horizontally, vertically, and diagonally adjacent nodes (a scale factor depending on the actual transmission parameters, namely the sensitivity at the FC receiver and the transmitter and receiver antenna gains) and $T_{p}^{(R L)}$ the time needed for packet transmission.

Overall, we can express the total consumed energy ${ }^{c}$ for the Radon-like CS-based approach as

$$
E_{\mathrm{RL}}=N_{\mathrm{TX}} G d^{2} T_{p}^{(\mathrm{RL})} .
$$

The packet duration $T_{p}^{(\mathrm{RL})}$ depends on the design of the selected sensing system. If the overall sensing framework is designed under the system constraint of having a fixed occupied bandwidth $B$, the packet duration time will be evaluated as $T_{p}^{(\mathrm{RL})}=L / B_{\mathrm{RL}}$ with $B_{\mathrm{RL}}=B$.

This approach is suited to an application scenario where the bandwidth devoted to inter-sensor communications is fixed in advance. Other possible system constraints concern the time interval during which a whole set of measurement is acquired. In this case, the time for refreshing of the measurement is fixed to $T_{c}$. Stemming on such a design constraint, the packet duration time needed to assure that the measurements are collected within a maximum time of $T_{c}$ is written as follows:

$$
T_{p}^{(\mathrm{RL})} \leq \frac{T_{c}}{N_{\mathrm{TS}}}
$$

Under this setting, the minimum occupied bandwidth, defined as the packet length in bits $L$ over the packet transmission time, for the Radon-like approach is evaluated as

$$
B_{\mathrm{RL}}=\frac{L}{T_{p}^{(\mathrm{RL})}} \geq \frac{L}{T_{c}} \delta_{P} \cdot \sqrt{N} .
$$

The relation providing the energy consumption in (15) can then be exploited either by considering an assigned packet time arising from a system bandwidth constraint or by assuming a given sensing rate $1 / T_{c}$. In the latter case, the packet duration is evaluated as in (16) so that the consumed energy reads as follows:

$$
E_{\mathrm{RL}}=\frac{\gamma_{P}}{\delta_{P}} T_{c} G d^{2} \cdot \sqrt{N} .
$$

Such system design choices should be carefully taken into consideration when comparing energy consumption of different schemes possibly comprising different numbers of single-hop transmissions $N_{\mathrm{TX}}$.

When performing numerical simulations, we have considered both the two aforementioned cases, namely:

- Fixed packet duration for the different compared schemes: this corresponds to a fixed system bandwidth constraint (cfr. results in Figure 6).

- Fixed sensing procedure duration $T_{c}$ : this corresponds to different packet durations (cfr. results in Figure 7).

\subsection{RS efficiency}

We now present a few results on the energy and bandwidth consumption of the approaches proposed in [10]. Therein, a RS procedure, allowing only a randomly chosen subset of sensors to acquire the measurement, is coupled with both a TDMA scheme and a random access scheme.

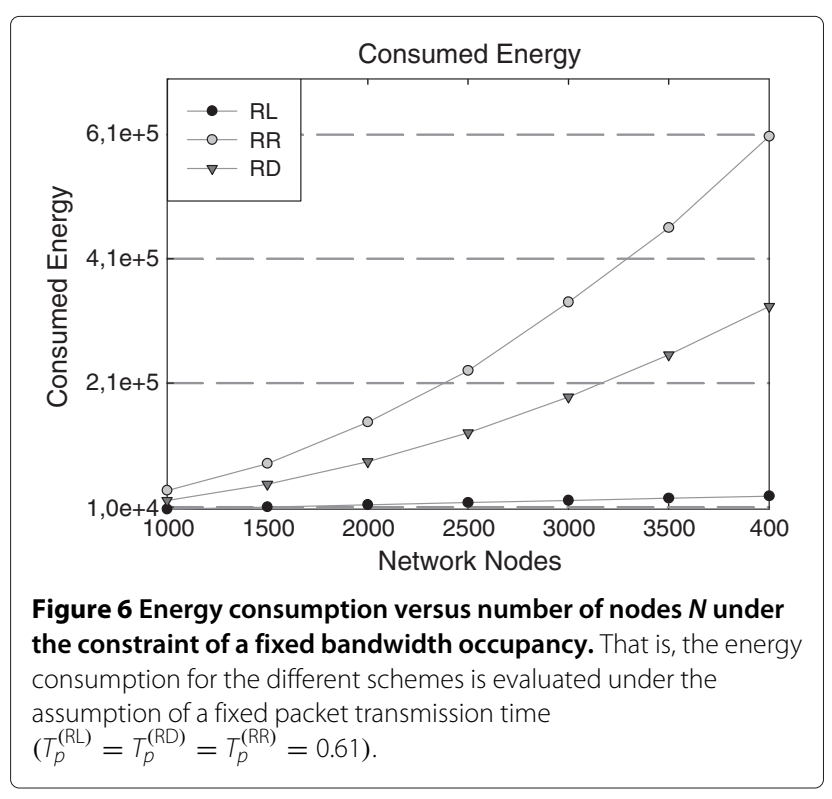




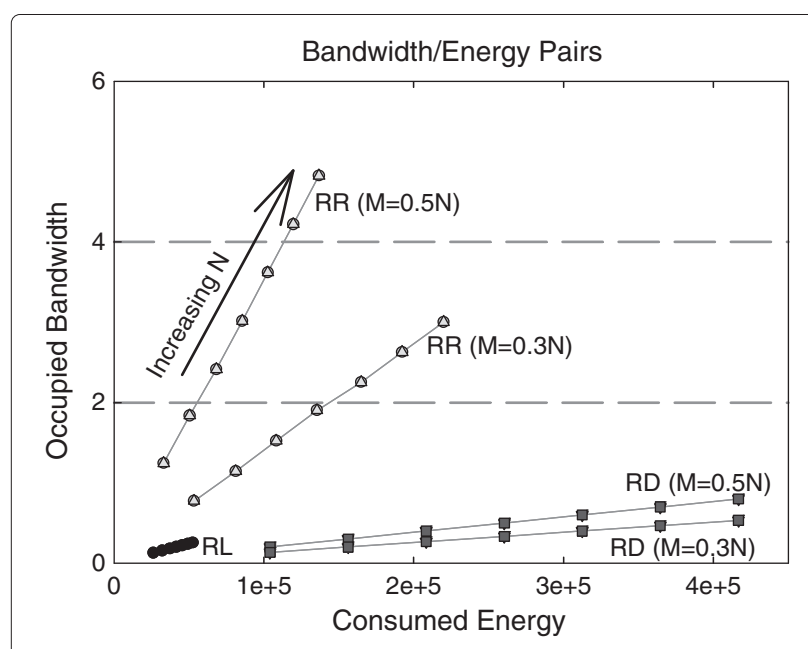

Figure 7 Energy/bandwidth pairs.

Herein we elaborate on these results and add a few details. With respect to the computation in [10], where the energy consumption for each sensor to transmit to the network sink is approximated by a constant, here we explicitly take into account the dependence of the energy with respect to (w.r.t.) the spatial sensor location. Secondly, therein an approximate relation is established between two key system parameters, namely (1) the minimal fraction of sensing data that must be correctly received at the sink to allow CS reconstruction and (2) the minimal bandwidth. Herein we extend the relation to different ranges of sensing probability, better suited to RS of a spatially sparse field.

In the RS/deterministic access (RD) scheme, the FC randomly chooses a set of $M$ sensors, $M$ being a sufficient number of measurements for satisfactory field reconstructions and broadcasting the addresses of eliged nodes through the network. The selected nodes acquire the measurements and transmit their readings to the FC via a TDMA deterministic access scheme. As in this scheme only $M$ nodes need to share the TDMA frame, the packet transmission time is $T_{p}^{(\mathrm{RD})}=T_{c} / M$. Consequently, the occupied bandwidth for the RD scheme is $B_{\mathrm{RD}}=M \frac{L}{T_{c}}$.

In order to evaluate the consumed energy, let us consider the set $\mathcal{C}_{M ; N}$ collecting all the possible configurations of $M$ out of $N$ nodes. The energy consumption of a given configuration $c \in \mathcal{C}_{M ; N}$ of $M$ is expressed as $E^{(c)}=$ $\sum_{k_{c}} G d_{k_{c}}^{2} T_{p}^{(\mathrm{RD})}$ where the sum over the index $k_{c}$ spans the $M$ sensors within the combination $c$. Thereby, the energy consumption of each combination depends on the distances of the $M$ nodes from the FC. In this respect, we evaluate the energy consumption of the RD scheme as the average over all the possible combinations: $E_{\mathrm{RD}}=$ $\frac{1}{K_{M ; N}} \sum_{c \in \mathcal{C}_{M ; N}} \sum_{k_{c}} G d_{k_{c}}^{2} T_{p}^{(\mathrm{RD})}$ where $K_{M ; N}=\left(\begin{array}{c}N \\ M\end{array}\right)$ is the cardinality of $\mathcal{C}_{M ; N}$. We recognize that in the overall sum over the $K_{M ; N}$ combinations, the energy spent by each and every network sensor appears in $\left(\begin{array}{c}N-1 \\ M-1\end{array}\right)$ terms, corresponding to the combinations it belongs to. Therefore, denoting $N_{1} \stackrel{\text { def }}{=} \alpha_{1} \sqrt{N}, N_{2} \stackrel{\text { def }}{=} \alpha_{2} \sqrt{N}$, the above sum can be rewritten as

$$
E_{\mathrm{RD}}=M \frac{\alpha_{1} \alpha_{2}\left(\alpha_{1}^{2}+\alpha_{2}^{2}\right)}{48} N T_{p}^{(\mathrm{RD})} .
$$

The energy consumption and occupied bandwidth performance of the scheme in [10] need to be addressed in a slightly different way w.r.t. the previous cases. CS theoretic results determine the number $M$ of measurements needed at the FC to correctly restore the sensed field; within an observation time, this constraint corresponds to a required percentage $q_{s}$ of correctly received samples at the FC. Because of possible collisions, the required percentage $q_{s}$ needed at the FC does not translate straightforwardly into a sensing probability $p_{s}$. In [10], the authors establish a relationship among the sensing probability $p_{s}$ and the probability $q_{s}$ of correct packet reception and demonstrate that, given $q_{s}$, a minimum bandwidth $B$ is required in order to assure that a feasible value of $p_{s}$ exists. Thereby, if the available bandwidth is not accurately dimensioned, small values of $p_{s}$ do not provide enough measurements at the $\mathrm{FC}$, whereas large values of $p_{s}$ cause too many collisions.

Besides, in [10], the authors provide an expression, standing for small values of $q_{s}$, of the minimum bandwidth as a function of the desired $q_{s}$. Following the guidelines in [10], we have extended such results to accommodate for large values of $q_{s}$, too. Specifically, we came up with the relation $B_{\mathrm{RR}}^{\min }=\frac{L}{T_{c}}\left(-2 N\left[\ln q_{s}\right]^{-1}+1\right) \quad$ if $\quad q_{s} \geq e^{-1}$. The RS settings are therefore assigned as follows. Firstly, the desired $q_{s}$ is fixed according to the reconstruction quality constraints. Secondly, the minimum needed bandwidth is evaluated. Finally, the selected bandwidth value is employed to derive the needed sensing probability $p_{s}[10]$. With these positions, the packet transmission time $T_{p}^{(\mathrm{RR})}$ is determined from the employed bandwidth as $T_{p}^{(\mathrm{RR})}=$ $L / B_{\mathrm{RR}}^{\min }$. Besides, the energy consumption is determined by the value of $p_{s}$; the average network consumed energy is evaluated as

$$
E_{\mathrm{RR}}=\frac{p_{s}}{24} N^{2} T_{p}^{(\mathrm{RR})} .
$$

\subsection{Further remarks}

Before turning to the numerical performance evaluation, a few remarks are in order. The above analysis has pointed out that the consumed energy and allocated bandwidth adopting the Radon-like CS scheme grow only with the square root $\sqrt{N}$ of the network size, whereas those of selected state-of-the-art approaches vary with 
the power $N$. The impact of these trends on energy consumption depends on all system parameters and mostly on $p_{s}$. For a spatially sparse field, where $p_{s}$ and consequently $q_{s}$ tend to be high, since a large fraction of sensors shall transmit their values using RS with random access to allow proper reconstruction, a random sensing scheme is prone to exhibit a large energy consumption and bandwidth occupancy. The Radon-like CS scheme then yields a reduced energy consumption for each node, as well as a parsimonious bandwidth use for collecting data over the entire grid. The gain is more and more evident as the network size (i.e., the covered area) increases.

Let us point out that, on non-spatially sparse fields, the conditions for accurate reconstruction, e.g., the values of $p_{s}$ and $P$, may differ, leading to different relative performances. The investigation of this issue is left for further studies.

The actual gain in terms of energy and bandwidth depends on the constants $\gamma_{P}, \delta_{P}$ which grow with the number of considered projections. The advantages of the Radon-like CS scheme are expected to be evident on spatially sparse signals, where low values of $P$ (e.g., $P=3$ ) enable reconstruction, whereas the RS data gathering algorithm requires a high percentage of samples to reach the FC in order to achieve satisfying reconstruction results [18].

As far as the medium access scheme is concerned, the herein-devised Radon-like CS scheme is realized via a TDMA technique, just as the RD scheme. Therefore, it implies an effort of synchronization and scheduling. Nonetheless, different data gathering procedures can be envisaged realizing the Radon-like CS using a random access criterion. This issue is left for further studies. Finally, these results depend on the peculiar structure of the Radon-like sensing matrix $\Phi_{R}$ and, although derived for a particular data gathering algorithm, can be generalized to different Radon-like CS measurement computation schemes.

\section{Numerical simulations}

In this section, we analyze the performance of our Radonlike CS scheme both in terms of reconstruction accuracy and of employed network resources. Firstly, in Section 7.1 we present a few results showing that an accurate reconstruction of a spatially sparse signal can be achieved by the Radon-like CS using a feasible number of Radonlike projections. Secondly, in Section 7.2 we demonstrate the energy and bandwidth gain achievable when adopting the Radon-like CS scheme in a WSN. For fair comparison of the different sensing techniques, we consider their performances under different system parameter settings, respectively corresponding to the case when each of the sensing system is operating at its minimal bandwidth and to the case of packet duration $T_{p}$ fixed for all the schemes. A comprehensive representation of the bandwidth-energy pairs of the different sensing systems is then shown versus the network dimensionality. Finally, we present a few results assessing the performance of Radon-like CS sampling on real-world data.

\subsection{Radon-like CS reconstruction accuracy}

With reference to the network model in Section 2, we consider a WSN made up by a square grid of $N=$ $64 \times 64=4,096$ sensors. The sensed field $z\left[n_{1}, n_{2}\right]$ is built up by seven repetitions of an elementary $5 \times 5$ pattern, differently scaled by factors in the range $(0.5-$ $0.85]$. Figure 8 a describes an example of the field $z\left[n_{1}, n_{2}\right]$. This field adheres to the so-called pulse stream signal model described in [25], and Algorithm 2 presented therein is employed for computing the reconstructed field $\hat{z}\left[n_{1}, n_{2}\right]$.

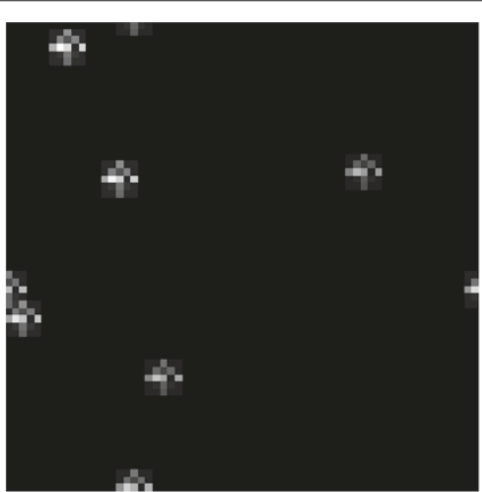

a)

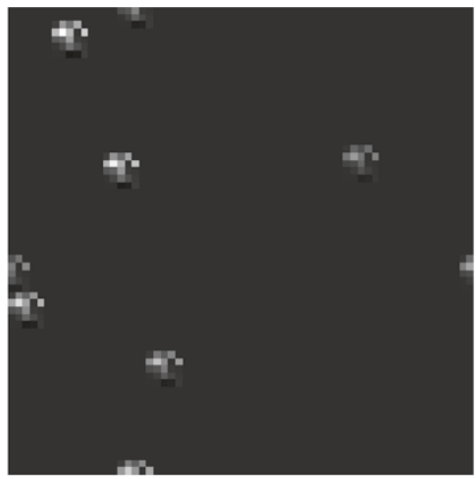

b)

Figure 8 Original field $z\left[\boldsymbol{n}_{1}, \boldsymbol{n}_{2}\right]$ (a) and reconstructed field $\hat{z}\left[\boldsymbol{n}_{\mathbf{1}}, \boldsymbol{n}_{\mathbf{2}}\right]$ (b) after sensing. Reconstruction was performed according to the Radon-like CS with $P=3$ projections. 
We have first evaluated the reconstruction accuracy of the Radon-like CS scheme, using different number $P$ of projections, under the assumption of noise-free observations. Specifically, we have tested the reconstruction accuracy when only projections along rows and columns of the network grid are considered $(P=2, M=128)$, when projections along the rows, columns, and the $\pi / 4$ oriented diagonal of the grid are considered $(P=3$, $M=255)$, and finally, also when projections along the $3 \pi / 4$-oriented diagonal are considered $(P=4$, $M=382$ ).

Table 1 reports the mean square error (MSE) of the reconstructed field $\hat{\mathbf{z}}$, i.e., $\operatorname{MSE}=\frac{1}{N}(\mathbf{z}-\hat{\mathbf{z}})^{\mathrm{T}}(\mathbf{z}-\hat{\mathbf{z}})$, achieved after 20 iterations of the reconstruction Algorithm 2 and averaged over ten runs corresponding to different pulse locations. Results in Table 1 show the effectiveness of the Radon-like CS scheme in sensing a sparse field with a restrained number of measurements. To visually assess the Radon-like CS scheme reconstruction accuracy, we show in Figure $8 \mathrm{~b}$ the reconstructed field $\hat{z}\left[n_{1}, n_{2}\right]$ obtained by measuring the field in Figure $8 \mathrm{a}$ with $P=3$ projections.

For the sake of comparison, we have also evaluated the reconstruction accuracy obtained by the RS [10] scheme under the same experimental settings. In Table 1 we recognize that, for the selected range of measurements $(M \leq 382)$, the RS does not allow capturing the sparse nature of the sensed field. To obtain the same reconstruction accuracy of the Radon-like CS scheme, namely a MSE equal to 0.0036 , the RS requires to be run with a number of measurements $M \simeq 2,500 \approx$ $50 \% N$.

Similar results have been obtained by scaling the number of network nodes to $N=80 \times 80=6,400$ and considering a sensed field $z\left[n_{1}, n_{2}\right]$ composed by eight repetitions of the elementary pulse. The MSE of the Radonlike CS scheme, averaged over ten runs, is reported in Table 2. Also, in this case, the MSE obtained by the Radonlike CS scheme with $P=\{2,3,4\}$, corresponding to $M=$ $\{160,319,478\}$ measurements, proves to exhibit satisfactory reconstruction quality. For the sake of comparison, we observe that in these experiments, $M=3,500$ were

\begin{tabular}{|c|c|c|c|}
\hline \multicolumn{4}{|l|}{ Parameter } \\
\hline M & 128 & 255 & 382 \\
\hline P & 2 & 3 & 4 \\
\hline$\vartheta_{p}$ & $0, \pi / 2$ & $\vartheta_{p}=0, \pi / 2, \pi / 4$ & $\vartheta_{p}=0, \pi / 2, \pm \pi / 4$ \\
\hline Radon-like CS - MSE & 0.0056 & 0.0019 & 0.0011 \\
\hline RS - MSE & 13.3 & 7.604 & 4.721 \\
\hline
\end{tabular}

Table 2 Reconstruction accuracy (MSE) obtained by Radon-like CS and RS schemes $(N=80 \times 80=6,400)$ for different numbers of measurements

\begin{tabular}{lccc}
\hline Parameter & \multicolumn{3}{c}{} \\
\hline$M$ & 160 & 319 & 478 \\
$P$ & 2 & 3 & 4 \\
$\vartheta_{p}$ & $0, \pi / 2$ & $\vartheta_{p}=0, \pi / 2, \pi / 4$ & $\vartheta_{p}=0, \pi / 2, \pm \pi / 4$ \\
Radon-like CS-MSE & 0.0045 & 0.00152 & 0.000901 \\
RS - MSE & 8.602 & 6.472 & 4.506 \\
\hline
\end{tabular}

needed by the RS to achieve analogous performance, namely a MSE equal to 0.006231 .

Finally, we have tested the Radon-like reconstruction accuracy when noisy acquisition is considered so that the CS measurements can be modeled as

$$
\mathbf{y}=\Phi \mathbf{z}+\mathbf{n}
$$

where the vector $\mathbf{n}$ gathers samples of white zero mean Gaussian noise with variance $\sigma_{n}^{2}$. In Table 3 we report the reconstruction accuracy obtained when acquiring with $N=64 \times 64=4,096$ sensors a field composed by seven patterns via the Radon-like CS scheme for different values of $\sigma_{n}^{2}$. Results in Table 3 show how the presence of noise in the acquisition process does not severely affect the reconstruction performance of the Radon-like CS approach.

To recap, the above results show that, as a rule of thumb, Radon-like CS requires $M \approx P \sqrt{N}$ measurements for representing a spatially sparse field, whereas the RS requires a large percentage of the measurements $M_{\mathrm{RS}} \approx \alpha N$ to be correctly received (e.g., $P=3$ and $\alpha=50 \%$ in the above experiments). Overall, the Radon-like CS scheme allows sensing and reconstructing of a spatially sparse field with far less measurement w.r.t. state-of-the-art techniques such as the RS presented in [10].

\subsection{Radon-like CS efficiency}

We now show that, besides using a restrained number of measurements, the Radon-like CS presents significant advantages in terms of energy and bandwidth needed to

Table 3 Reconstruction accuracy (MSE) obtained by the Radon-like CS, noisy measurements $(N=80 \times 80=6,400)$

\begin{tabular}{lccc}
\hline Parameter & \multicolumn{3}{l}{} \\
\hline M & 128 & 255 & 382 \\
$P$ & 2 & 3 & 4 \\
$\vartheta_{p}$ & $0, \pi / 2$ & $\vartheta_{p}=0, \pi / 2, \pi / 4$ & $\vartheta_{p}=0, \pi / 2, \pm \pi / 4$ \\
Radon-like CS-MSE & 0.0065 & 0.0034 & 0.0033 \\
for $\sigma_{n}^{2}=0.5$ & & & \\
Radon-like CS-MSE & 0.007 & 0.0039 & 0.0036 \\
for $\sigma_{n}^{2}=0.7$ & & & \\
\hline
\end{tabular}


disseminate sensor readings towards the FC. We evaluate the performance of Radon-like CS data gathering scheme (RL) by evaluating the consumed energy and occupied bandwidth under the following assumptions:

(a1) The number of measurements chosen is large enough to yield a satisfactory reconstruction accuracy, quantified by a $\mathrm{MSE} \leq 10^{-3}$.

(a2) The coherence time of the sensed field is fixed to $T_{c}=2,500 s$.

(a3) The dimension of the transmitted packet is set to $L=1 \mathrm{~Kb}$.

With reference to the experimental setting described in Section 7.1, condition (a1) implies $P=3$ projections. We start by computing the energy consumption under the assumption that the occupied bandwidth and, consequently, the packet transmission time $T_{p}=L / B$ are the same for the different schemes.

Let us remark that, under this setting corresponding to a system design choice of having a fixed occupied bandwidth, each scheme requires a different number of transmissions in order to let the FC acquire the needed measurements. Thereby, the overall process of sensing is accomplished in different time intervals by the different data gathering techniques. In these experiments, we have set $T_{p}^{(R L)}=T_{p}^{(\mathrm{RD})}=T_{p}^{(\mathrm{RR})}=T_{p}=0.61$. Figure 6 reports the energy consumed for different numbers of network sensors $N$. We recognize that employing the $\mathrm{RL}$ scheme drastically reduces the energy consumed by the data gathering algorithm.

We then proceed to compute for the minimum occupied bandwidth required by the RL scheme to guarantee correct sensing and reconstruction of a spatially sparse field under the constraint of a given refresh time $T_{c}$. Figure 9

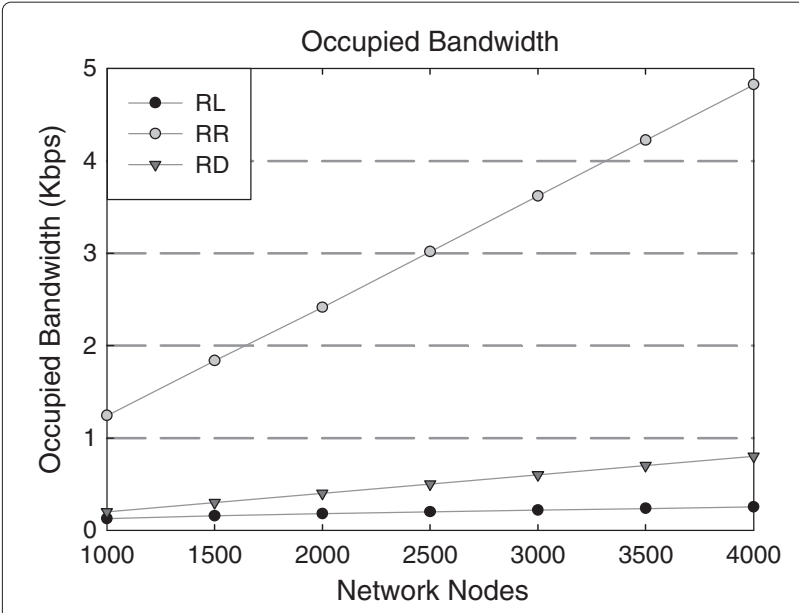

Figure 9 Minimum bandwidth occupancy versus the number of nodes $N$. plots the occupied bandwidth evaluated according to (17) versus the network size in terms of the number of sensors.

For the sake of comparison, in the same figure, we also report the performance of RS, implemented using both a deterministic access scheme (RD in the legend) and the random access scheme ( $R R$ in the legend) described in [10]. The occupied bandwidth is computed according to the analysis in Section 6 for the RL, RD, and RR scheme, respectively, under the same assumptions (a1) to (a3). These settings imply $p_{s}=0.5$ for the RD scheme and $q_{s}=0.5$ for the RR one.

The bandwidth employed for the Radon-like data gathering scheme is significantly reduced w.r.t. the RS, both using deterministic and random access. The above results, obtained by considering different numbers of measurements and equal reconstruction accuracy for the three algorithms, are explained by the efficiency of the Radonlike data collection algorithm, exploiting only single-hop, parallel data transmission.

We have then pushed further the comparison to find under which conditions the Radon-like and the RS schemes equally perform. In doing so, we have evaluated the bandwidth occupied by the RD and RR schemes when only $10 \%$ of the sensor readings are required to be correctly received by the FC. Figure 10 reports these results showing that the RL scheme still favorably compares with the RR scheme while requiring just slightly higher bandwidth w.r.t. the RD scheme. Therefore, the RL scheme overcomes the RD and RR ones unless they use a very low percentage of the sensors' readings for field reconstruction.

Finally, we are interested in comparing the resource employed by the different schemes to accomplish the sensing process exactly at the same time. To reach this

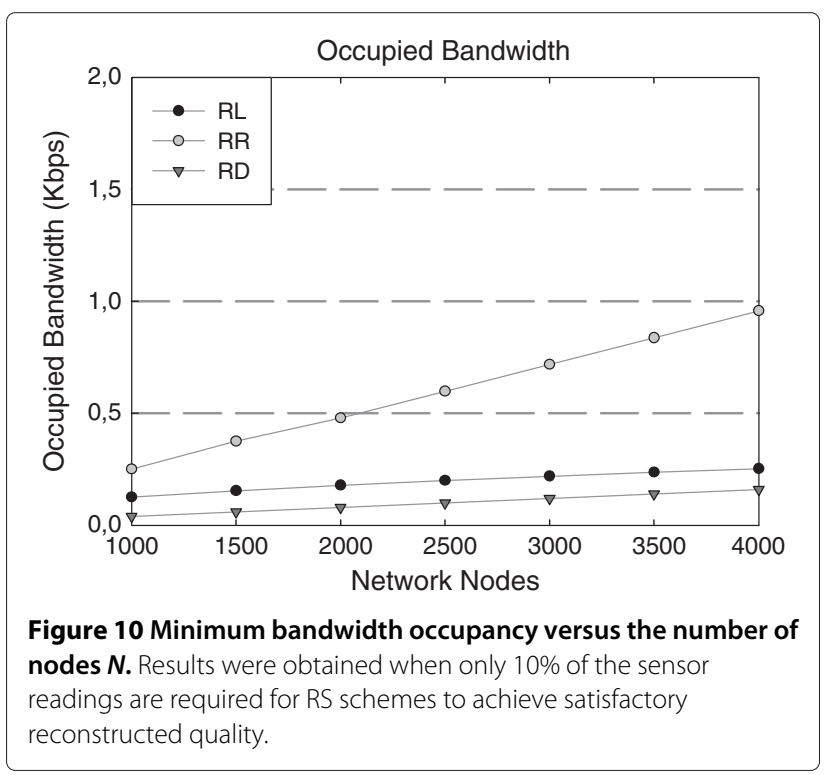


goal, as the number of single-hop transmissions varies from one scheme to another, the different schemes will have a different packet transmission time and hence will occupy a different bandwidth. In Figure 7, we illustrate the bandwidth-energy scatterplot of the RL, RR, and RD schemes for different network sizes. For RD and RR, we have considered two cases, that is, when $50 \%$ and $30 \%$ of the sensor readings are required at the FC for satisfactory field reconstruction.

The energy/bandwidth pairs draw different trajectories while the number of nodes $N$ increases. We clearly recognize a systematic energy and bandwidth saving of the RL scheme w.r.t. the competitors. Moreover, both the consumed energy and the occupied bandwidth exhibit far smaller variations with the number of network nodes than what happens with the RD and RR schemes, making the $\mathrm{RL}$ approach fully scalable in terms of network nodes.

\subsection{Radon-like CS performances on real data sets}

We now apply the Radon-like CS matrix to real-world data. Following the approach in [10], we resort to public oceanographic databases, namely the ones in [26]. Specifically, we have considered the compressive sensing acquisition of the 'Zonal Current' data at Monterey Bay on October 10, 2012. The Zonal Current data at the selected day are represented in Figure 11 (left). Let us highlight that the considered data are not spatially sparse. On the contrary, visual inspection of Figure 11 (left) shows how the field is represented by a pronounced pulse embedded in small values. Thereby, different approaches can be pursued. For instance, Radon-like sampling could be integrated with suitable basis transform to track for signal sparsity; this approach is left for further studies. Here we undertake a different procedure. Although the field is not strictly sparse as most of its values are indeed non-zero, we observe that it is yet true that most of the information conveyed by the field in Figure 11 (left) lies on its peaks. For the reader's convenience, we report in Figure 12 a histogram of the field values in Figure 11 (left), and in Figure 13, a histogram of the thresholded field value in Figure 11 (center) for $t=0.2$. In Figure 12, we have also indicated the thresholding value (black dashed line) and the mean value of the sensed field (red solid line).

Stemming from this observation, we employ the Radonlike CS scheme to acquire a thresholded version of the original field. We acquire the $(81 \times 81)$ sensed field for different values of the threshold $t$, using a Radon-like scheme with $P=3$. This scheme comprises projections along the rows, columns, and the $\pi / 4$-oriented diagonal of the grid, thus resulting in $M=323$. Then we employ the CoSaMP algorithm as the reconstruction procedure. The reconstructed image is finally compared to the original, not thresholded, field so as to evaluate the MSE with respect to the original image. In Table 4, we report the results obtained for different values of the threshold $t$, corresponding to different degrees of sparsity of the thresholded image. We also report in Table 4 the number of non-zero samples in the thresholded image.

The results in Table 4 confirm the effectiveness of the Radon-like scheme in enabling reconstruction of the spatially sparse nature of the sensed thresholded field, achieving MSE as low as 0.0036 for a high threshold value such as $t=0.2$. As the thresholding factor, the number of non-zero coefficients in the thresholded image increases, making the Radon-like approach less effective. We would like to emphasize that application of Radon-like sensing matrices to non-sparse data could still be possible by taking into account suitable sparsifying basis, which is left for further studies.

In Figure 11 (right), we report the reconstructed thresholded field after 15 iterations of the CoSaMP algorithm for $t=0.2$; the corresponding original thresholded image is reported in Figure 11 (center). Visual inspection of Figure 11 (right) shows how, in spite of the
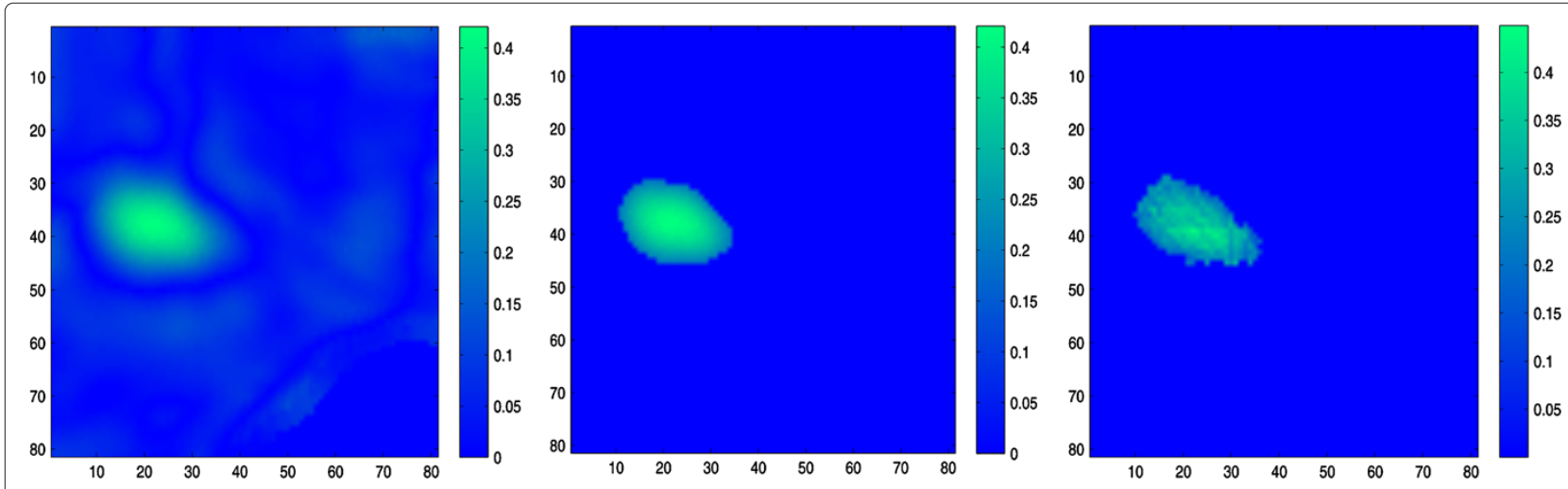

Figure 11 Compressive sensing acquisition of the Zonal Current data. Zonal Current field sensed at Monterey Bay on October 10, 2012 (left), thresholded field sensed $(t=0.2)$ (center), and reconstructed thresholded field sensed obtained by running the CoSaMP algorithm over the CS measurements obtained by the Radon-like approach for $P=3, M=323, t=0.2$ (right). 


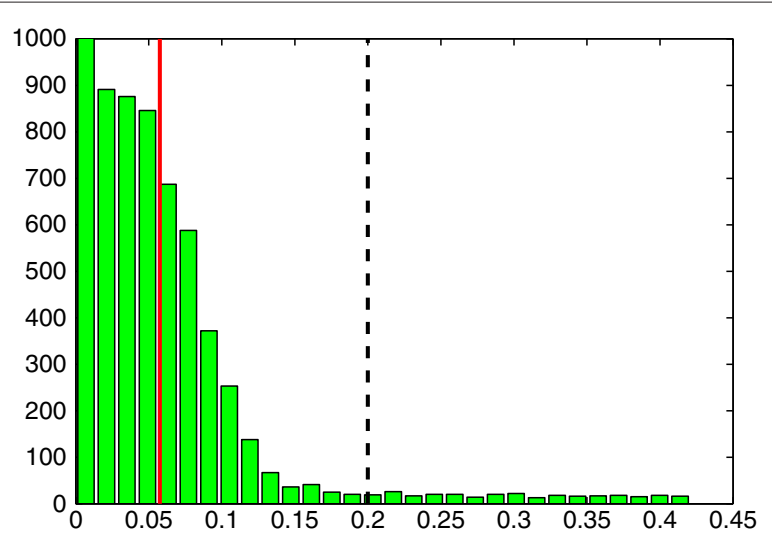

Figure 12 Histogram of the sensed field values. The black dashed line represents the thresholding value, and the red solid line represents the mean value of the sensed field.

reduced number of measurements and of the restrained energy consumption, the Radon-like scheme is capable of capturing the spatially sparse nature of the reconstructed field. Finally, the approach outlined herein could be enforced by taking into account suitable padding of the data samples that are under the threshold, so as to substitute the zero entries obtained by the reconstruction algorithm with a specific non-zero value, e.g., their spatial mean, thereby reducing the observed MSE.

\subsection{Final remarks}

As a final remark, we observe that energy and bandwidth gain yielded by the Radon-like approach is directly based upon the favorable matching between the Radonlike matrix structure and the spatially sparse structure of the sensed field. Based on these results, we envisage a twofold extension of our work, namely:

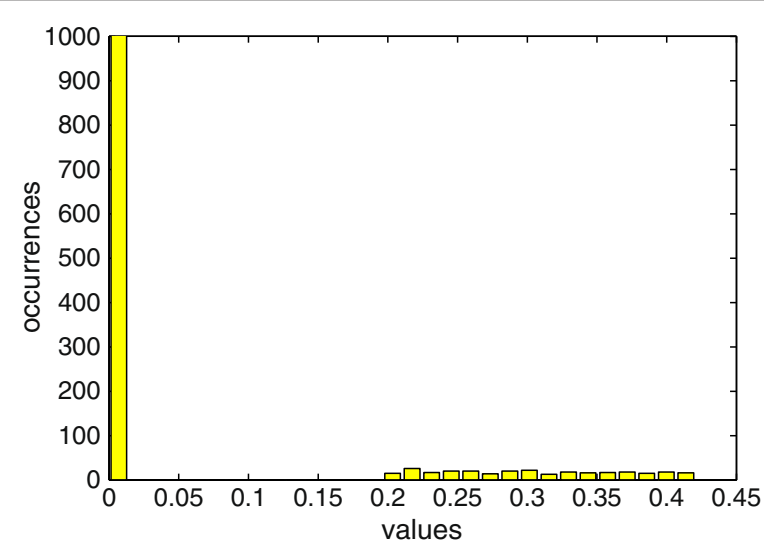

Figure 13 Histogram of the thresholded field values. The threshold has been set to $t=0.2$.
Table 4 Reconstruction performance in a real-data scenario (Zonal Current at Monterey Bay [26]) for different thresholding values

\begin{tabular}{lccc}
\hline $\boldsymbol{t}$ & $\boldsymbol{S}$ & $\begin{array}{c}\text { MSE against original } \\
\text { field }\end{array}$ & $\begin{array}{c}\text { MSE against } \\
\text { thresholded field }\end{array}$ \\
\hline 0.1 & 826 & 0.003 & 0.0015 \\
0.125 & 485 & 0.0031 & $8.20 \times 10^{-4}$ \\
0.15 & 386 & 0.0033 & $6.67 \times 10^{-4}$ \\
0.175 & 319 & 0.0035 & $5.89 \times 10^{-4}$ \\
0.2 & 285 & 0.0036 & $5.59 \times 10^{-4}$ \\
\hline
\end{tabular}

Results refer to Radon-like CS with $P=3, M=323$.

- In accounting an irregular sampling grid

- In exploiting non-straight sampling path.

These conditions may be encountered, for instance, in vehicular networks or citizen sensing networks [27], where the disposition of the nodes are far from being regular, and the sampling path should adapt to the routing paths, which in turn basically depend on the street and building disposition. To sum up, we consider this contribution as an intermediate step towards finding a general relation between the compressive sensing of a finite innovation rate signal and its realization by efficient routing algorithms in a realistic WSN scenario.

\section{Conclusions}

In this paper, we have addressed the efficient compressive sampling of spatially sparse signals in sensor networks. Specifically, we have introduced a peculiar CS sampling scheme for spatially sparse bidimensional signals. We have analytically demonstrated that our scheme satisfies the theoretical conditions required for CS signal reconstruction. Then after devising a distributed data gathering scheme for collecting of the CS measurements in a WSN, we have characterized the scheme both in terms of consumed transmission energy and occupied bandwidth. The scheme outperforms state-of-the-art schemes for spatially sparse fields, and it represents an intermediate step towards the definition of routing procedures well suited to the characteristics of the signal a realistic sensor network is faced with.

\section{Endnotes}

aThe coherence time $T_{c}$ is defined as the time interval over which the process almost de-correlates in time.

bormally, we obtain the $\vartheta_{p}$-radiant clockwise-rotated version of the image $z\left[n_{1}, n_{2}\right]$ by regular sampling of the rotated field

$s^{\left(\vartheta_{p}\right)}(x, y ; t)=s\left(x \cos \vartheta_{p}+y \sin \vartheta_{p}, x \sin \vartheta_{p}-y \cos \vartheta_{p} ; t\right)$.

'We also approximate herein the inter-sensor distance in the diagonal direction to $d$. 


\section{Appendix 1 :RIP property of the Radon-like measurements matrix}

Property Let us assume that the entries in the matrix $\Phi_{R}$ are either deterministically set to zero or drawn from i.i.d. zero mean Gaussian random variables with equal variance $\sigma_{\varphi}^{2}=1 / P$; the following concentration inequality stands:

$$
\operatorname{Pr}\left\{\left|\mathcal{E}_{y}-\mathcal{E}_{z}\right| \geq \delta\right\} \leq \epsilon
$$

provided that $P \geq 2 K^{2} C_{2}^{2} \log (2 / \epsilon) / \delta^{2}, C_{2}$ being a suitable constant.

Demonstration The condition in (20) can be demonstrated as follows. Let us consider the sample energy $\mathcal{E}_{y}$ of the measurements. $\mathcal{E}_{y}$ being a sample moment, we invoke here its asymptotical normal distributions [28]. Although this hypothesis is not necessary for the RIP to stand, it allows us to straightforwardly evaluate the minimal number of projections $P$ required for $K$-sparse field reconstruction, and therefore we retain it in the following.

The Chernov bound [29] for a normal random variable establishes that the probability that the random variable differs from its mean is limited by a term exponentially decaying with its variance. By applying the Chernov bound to the random variate $\mathcal{E}_{y}$, we obtain

$$
\operatorname{Pr}\left\{\left|\mathcal{E}_{y}-\mathrm{E}\left\{\mathcal{E}_{y}\right\}\right| \geq \delta\right\} \leq 2 \cdot \exp ^{-\delta^{2} / 2 \sigma_{\mathcal{E}_{y}}^{2}}
$$

Thereby, in order to demonstrate (12), it suffices to show that $\mathrm{E}\left\{\mathcal{E}_{y}\right\}=\mathcal{E}_{z}$ and $\operatorname{Var}\left\{\mathcal{E}_{y}\right\}=o(P)$ for the case under concern.

By definition we have

$$
\mathcal{E}_{y}=\sum_{p=0}^{P-1} \sum_{m=0}^{K(p)-1}\left(\sum_{i} \varphi_{m}^{(p)}[i] z^{\left(\vartheta_{p}\right)}[i, m]\right)^{2} .
$$

Let us denote by

$$
\begin{aligned}
y_{m}^{\left(\vartheta_{p}\right)} \stackrel{\text { def }}{=} \sum_{i} \varphi_{m}^{(p)}[i] z^{\left(\vartheta_{p}\right)}[i, m], p & =0 \ldots P-1, \\
m & =0, \ldots K(p)-1 .
\end{aligned}
$$

We recognize that $y_{m}^{\left(\vartheta_{p}\right)}$ are independent normal random variables, with zero mean and variance equal to

$$
\operatorname{Var} y_{m}^{\left(\vartheta_{p}\right)}=\sigma_{\varphi}^{2} \sum_{i}\left(z^{\left(\vartheta_{p}\right)}[i, m]\right)^{2}
$$

We then evaluate the expected value of $\mathcal{E}_{y}$ as follows:

$$
\mathrm{E}\left\{\mathcal{E}_{y}\right\}=\sum_{p=0}^{P-1} \sum_{m=0}^{K(p)-1} \operatorname{Var} y_{m}^{\left(\vartheta_{p}\right)} .
$$

Substituting (21) in (22) and recognizing that $\sum_{m} \sum_{i}\left(z^{\left(\vartheta_{p}\right)}[i, m]\right)^{2}=\mathcal{E}_{z}$, we have

$$
\mathrm{E}\left\{\mathcal{E}_{y}\right\}=\sigma_{\varphi}^{2} \cdot P \cdot \mathcal{E}_{z}
$$

Hence, by setting $\sigma_{\varphi}^{2}=1 / P$, we get $\mathrm{E}\left\{\mathcal{E}_{y}\right\}=\mathcal{E}_{z}$.
As far as the variance of $\mathcal{E}_{y}$ is concerned, we obtain

$$
\begin{aligned}
\operatorname{Var} \mathcal{E}_{y} & =\sum_{p=0}^{P-1} \sum_{m=0}^{K(p)-1} \operatorname{Var}\left(y_{m}^{\left(\vartheta_{p}\right)}\right)^{2} \\
& =\sum_{p=0}^{P-1} \sum_{m=0}^{K(p)-1}\left(\mathrm{E}\left\{\left(y_{m}^{\left(\vartheta_{p}\right)}\right)^{4}\right\}-\mathrm{E}\left\{\left(y_{m}^{\left(\vartheta_{p}\right)}\right)^{2}\right\}^{2}\right)
\end{aligned}
$$

Since the variables $y_{m}^{\left(\vartheta_{p}\right)}$ are zero mean normally distributed, their fourth-order moments satisfy

$$
\mathrm{E}\left\{\left(y_{m}^{\left(\vartheta_{p}\right)}\right)^{4}\right\}=3 \cdot\left(\operatorname{Var} y_{m}^{\left(\vartheta_{p}\right)}\right)^{2}
$$

so that we obtain

$$
\operatorname{Var} \mathcal{E}_{y}=2 \sum_{p=0}^{P-1} \sum_{m=0}^{K(p)-1}\left(\operatorname{Var} y_{m}^{\left(\vartheta_{p}\right)}\right)^{2}
$$

Observing that, for a $K$-sparse signal, the maximum value of $\sum_{i_{2}}\left(z^{\left(\vartheta_{p}\right)}\left[i_{2}, m\right]\right)^{2}$ is achieved in case of $K$ aligned pulses, we recognize that the following inequality stands:

$$
\begin{aligned}
\left(\operatorname{Var} y_{m}^{\left(\vartheta_{p}\right)}\right)^{2} & =\sigma_{\varphi}^{4} \sum_{p=0}^{P-1} \sum_{m=0}^{K(p)-1} \sum_{i_{1}}\left(z^{\left(\vartheta_{p}\right)}\left[i_{1}, m\right]\right)^{2} \\
& \cdot \sum_{i_{2}}\left(z^{\left(\vartheta_{p}\right)}\left[i_{2}, m\right]\right)^{2} \\
& \leq \sigma_{\varphi}^{4} \sum_{p=0}^{P-1} \sum_{m=0}^{K(p)-1} \sum_{i_{1}}\left(z^{\left(\vartheta_{p}\right)}\left[i_{1}, m\right]\right)^{2} \cdot K C_{2} \\
& =\sigma_{\varphi}^{4} P \cdot \mathcal{E}_{z} \cdot K C_{2}
\end{aligned}
$$

with $C_{2}=\max _{\left(n_{1}, n_{2}\right)} z\left[n_{1}, n_{2}\right]^{2}$.

Finally, we recognize that the variance $\operatorname{Var}\left\{\mathcal{E}_{y}\right\}$ is upper bounded by

$$
\operatorname{Var}\left\{\mathcal{E}_{y}\right\} \leq \frac{1}{P} \cdot K^{2} C_{2}^{2} .
$$

In (24), we recognize that the variance $\operatorname{Var}\left\{\mathcal{E}_{y}\right\}$ decays as $1 / P$. Furthermore, by comparing (24) and (12), we recognize that the RIP is verified provided that the number of projections $P$ satisfies

$$
P \geq 2 K^{2} C_{2}^{2} \log (2 / \epsilon) / \delta^{2} .
$$

The demonstration presented herein proves that the Radon-like CS matrix satisfies the RIP property in the spatial domain, i.e., under the assumption that the sparsifying basis is the canonical basis. The interested reader can find a proof of the RIP property for the Radon-like matrix in any orthonormal non-canonical basis in [30].

\section{Appendix 2 :Within WSN Radon-like projections' computation}

Let us consider a regular network composed by $N=N_{1} N_{2}$ sensors as in Figure 1 where, without loss of generality, we 
assume that $N_{1}$ and $N_{2}$ are odd valued, i.e., $N_{1}=2 \tilde{N}_{1}+1$, $N_{2}=2 \tilde{N}_{2}+1$, so as to identify a central column where the FC is located.

We discuss a simple suboptimal procedure to collect all the projections to the FC using a TDMA access scheme. Let us subdivide the network into four quadrants, and let us consider first the horizontal projections $p_{H}[m]$, obtained by accumulating randomly weighted values along the network rows. In each quadrant, the data gathering process starts at the outer nodes. The external node in each row measures the field, computes the product of the reading with a randomly selected coefficient, encodes this value in a packet of $L$ bits, and transmits it to the neighboring node in the horizontal direction. The neighboring node, once the packet from the outer node has been received, measures the field, multiplies the reading by the random coefficient, and sums it to the value received by the outer node. The overall process continues until the nodes in the central column are reached by the data flow and are then ready to propagate the projection values to the FC. Once the FC has received the horizontal projection values from the first quadrant, the same operations are serially performed in the three remaining quadrants. The projection values of each quadrant are therefore computed by evaluating partial sums and propagating them towards the nodes in the central column; then the projection values are transmitted to the $\mathrm{FC}$ via a multi-hop route along the central column.

Let us now evaluate the number of transmissions required to compute the horizontal projections. To collect the contributions within a network quadrant, we need the following:

- $\tilde{N}_{1}$ transmission to reach the central column for each of the $\tilde{N}_{2}+1$ rows

- $\sum_{l=0}^{\tilde{N}_{1}} l$ transmissions to propagate the projection value towards the $\mathrm{FC}$ along the central column

Accounting for the four quadrants, the overall number of transmissions for horizontal projection evaluation sums up to

$$
N_{\mathrm{TX}}^{(\pi / 2)}=\frac{N_{1}-1}{2}\left(2 N_{2}+N_{1}+3\right) .
$$

By denoting $N_{1} \stackrel{\text { def }}{=} \alpha_{1} \sqrt{N}, N_{2} \stackrel{\text { def }}{=} \alpha_{2} \sqrt{N}$, we can write

$$
N_{\mathrm{TX}}^{(\pi / 2)}=\left(\alpha_{1} \alpha_{2}+\frac{\alpha_{1}^{2}}{2}\right) N+\left(\alpha_{1}-\alpha_{2}\right) \sqrt{(}(N)-1.5 \text {. }
$$

Let us now evaluate the number of time slots in which the $N_{\mathrm{TX}}^{(\pi / 2)}$ transmissions can be performed. Since signaling occurs between adjacent nodes, the propagation of information on the different rows can be scheduled in parallel flows, provided that a suitable inter-row delay of $v_{0}$ time slots is introduced to prevent interference among neighboring nodes.
Let us sketch out a possible time scheduling for withinquadrant transmission, corresponding to the following gathering protocol:

- The data gathering starts at $t_{0}=0$, on the first row of the quadrant, i.e., the one comprising the FC. The outermost node transmits its randomly weighted sensed value in the first time slot. In the second time slot, the second node forwards the sum of the received data and its own randomly weighted sensed value. Similarly, each node updates and sends the received partial sum. Thereby, the FC retrieves the accumulation after $t_{f}^{1}=\tilde{N}_{1} T_{p}$, with $T_{p}$ being the duration of a time slot.

- On the second row, the transmission begins after $v_{0}$ time slots to avoid interference with the first-row transmission. Then $\tilde{N}_{1}$ time slots are needed for the partial sum to reach the central column, and one additional time slot is needed to reach the FC. The propagation ends at $t_{f}^{2}=\left(v_{0}+\tilde{N}_{1}+1\right) T_{p}$.

- On the $i$ th row, the transmission begins after $(i-1) \cdot v_{0}$ time slots, and the propagation ends at $t_{f}^{i}=\left(i \cdot v_{0}+\tilde{N}_{2}+i\right) T_{p}$.

- On the $\left(\tilde{N}_{2}+1\right)$-th row, transmission to the FC is accomplished at $t_{f}^{\tilde{N}_{2}+1}=\left(\tilde{N}_{2} v_{0}+\tilde{N}_{1}+\tilde{N}_{2}\right) T_{p}$.

For the sake of clarity, we report in Figure 14 a scheme summarizing the timing of the nodes' transmissions when computing the horizontal projections $p_{H}[m]$ within a quadrant of the network.

The FC is then able to collect all the horizontal projections in a quadrant after $N_{q}=\tilde{N}_{2} v_{0}+\tilde{N}_{1}+\tilde{N}_{2}$ time slots. If the quadrants are visited in a serial fashion, the overall number of time slots to compute the horizontal projections accounts for

$$
N_{\mathrm{TS}}^{(\pi / 2)}=4\left(\tilde{N}_{2} v_{0}+\tilde{N}_{1}+\tilde{N}_{2}\right) .
$$

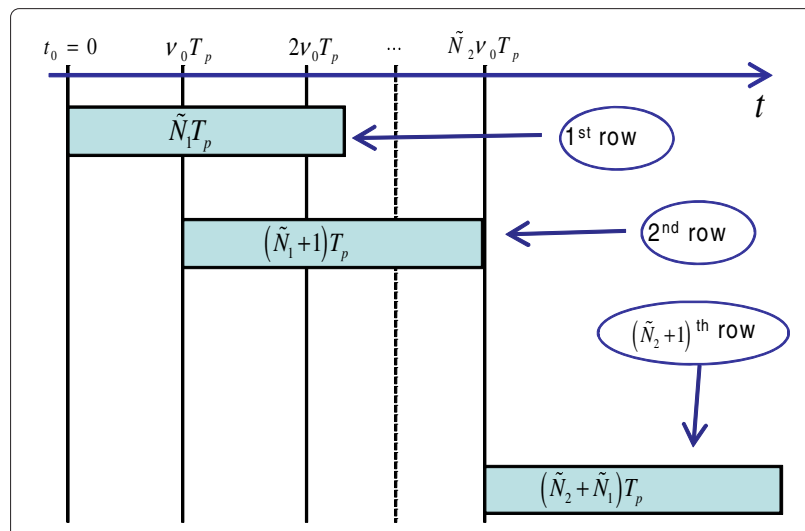

Figure 14 Data gathering algorithm: timing of the nodes' transmissions for within quadrant evaluation of $p_{H}[m]$. 
Again, by denoting $N_{1} \stackrel{\text { def }}{=} \alpha_{1} \sqrt{N}, N_{2} \stackrel{\text { def }}{=} \alpha_{2} \sqrt{N}$, we have

$$
N_{\mathrm{TS}}^{(\pi / 2)}=2 \sqrt{N}\left[\alpha_{2}+\alpha_{1}\left(1+v_{0}\right)\right]-\left(4+2 v_{0}\right) .
$$

The overall protocol for evaluating $p_{H}[m]$ is illustrated in Figure 4.

An important remark is in order. Despite its simplicity, this basic result highlights one of the major advantages of the Radon-like CS scheme. Since the Radon-like projections can be evaluated by means of information propagation on linear paths in the network, the number of single-hop transmissions vary with the product depth and width of the network grid, that is, with the network size $N$. On the other hand, since only single-hop transmissions are employed, the transmission can be parallelized, and the number of time slots required for computation of an assigned projection set $y^{\left(\vartheta_{p}\right)}[\mathrm{m}]$ varies with the sum of depth and width of the network grid, that is, with the square root of the network size $N$. This intrinsic behavior, which holds for different projections' directions, is the reason why the Radon-like CS scheme will be proven to be both energy- and bandwidth-efficient.

With slight modifications, the above described procedure can be extended to the case of differently tilted paths. For the sake of concreteness, we develop in the following the calculations for $\vartheta_{p}=0$ (vertical projections), $\vartheta_{p}=$ $\pm \pi / 4$ (diagonal projections), which have been considered in the simulations described in this paper.

Vertical projections $p^{(0)}[m]$ can be computed using the same protocols adopted for the horizontal projections so that we have

$$
\begin{aligned}
& N_{\mathrm{TX}}^{(0)}=\frac{N_{2}-1}{2}\left(2 N_{1}+N_{2}+3\right) \\
& N_{\mathrm{TS}}^{(0)}=4\left(\tilde{N}_{1} v_{0}+\tilde{N}_{2}+\tilde{N}_{1}\right)
\end{aligned}
$$

or equivalently

$$
\begin{aligned}
& \left.N_{\mathrm{TX}}^{(0)}=\left(\alpha_{2} \alpha_{1}+\frac{\alpha_{2}^{2}}{2}\right) N+\left(\alpha_{2}-\alpha_{1}\right) \sqrt{(} N\right)-1.5 \\
& N_{\mathrm{TS}}^{(0)}=2 \sqrt{N}\left[\alpha_{1}+\alpha_{2}\left(1+v_{0}\right)\right]-\left(4+2 v_{0}\right) .
\end{aligned}
$$

If only horizontal and vertical projections are considered within the Radon-like CS scheme, stemming from (25) and (27), we can evaluate the overall number of transmissions needed to propagate data towards the FC as

$$
N_{\mathrm{TX}}^{(0, \pi / 2)}=\left(2 \alpha_{1} \alpha_{2}+\frac{\alpha_{1}^{2}+\alpha_{2}^{2}}{2}\right) N-3
$$

where we recognize how the required number of transmissions grows linearly with the number of network nodes

$$
\begin{aligned}
& N_{\mathrm{TX}}^{(0, \pi / 2)} \approx \gamma_{2} N \\
& \gamma_{2}=\left(2 \alpha_{1} \alpha_{2}+\frac{\alpha_{1}^{2}+\alpha_{2}^{2}}{2}\right) .
\end{aligned}
$$

Regarding the number of time slots needed to collect the sensors' readings, referring to (27) and (28), we have

$$
N_{\mathrm{TS}}^{(0, \pi / 2)}=\left(\alpha_{1}+\alpha_{2}\right)\left(4+2 v_{0}\right) \sqrt{N}-\left(8+4 v_{0}\right)
$$

where we recognize how the required number of time slots grows with the squared root of the network size:

$$
\begin{aligned}
& N_{\mathrm{TS}}^{(0, \pi / 2)} \approx \delta_{2} \sqrt{N} \\
& \delta_{2}=\left(\alpha_{1}+\alpha_{2}\right)\left(4+2 \nu_{0}\right) .
\end{aligned}
$$

For the type of diagonal projections $r_{D}[m]$ concerned, the procedure is similar to the previous case in the sense that the gathering scheme is aimed at propagating the projection values from the outer row and column of the quadrant to the FC by first reaching the nodes in the inner row and column. We serially perform the accumulations in each quadrant, as described for the horizontal projection, along the following paths:

(p1) All the $\pi / 4$-oriented paths that originate from the nodes lying along the outer row of the quadrant and that reach the FC through either the central row or the central column (solid arrows in Figure 5)

(p2) All the $\pi / 4$-oriented paths that originate from the nodes lying along the outer column of the quadrant and that reach the FC through either the central row or the central column (dashed arrows in Figure 5).

The paths (p1) and (p2) both exhibit a length of at most $\max \left\{\tilde{N}_{1}, \tilde{N}_{2}\right\}$. Each of the $\tilde{N}_{1}+\tilde{N}_{2}+1$ projections are composed by at most $\max \left\{\tilde{N}_{1}, \tilde{N}_{2}\right\}$ transmissions, so that the overall number of needed transmissions sums up to

$$
\begin{aligned}
N_{\mathrm{TX}}^{(\pi / 4)} & =\left(N_{1}+N_{2}\right)\left(\max \left\{N_{1}, N_{2}\right\}-1\right) \\
& =\left(\alpha_{1}+\alpha_{2}\right) \max \left\{\alpha_{1}, \alpha_{2}\right\} N-\left(\alpha_{1}+\alpha_{2}\right) \sqrt{N} .
\end{aligned}
$$

If the quadrant is processed so that we firstly perform the accumulations along the paths in (p1) starting at $t_{0}=$ 0 and secondly perform the accumulations along the paths in (p2), then

- The first projection value along the paths in (p1) reaches the FC at $t_{f}^{1}=\tilde{N}_{2} T_{p}$.

- The last projection value along the paths in (p1) reaches the $\mathrm{FC}$ at most at $t_{f}^{\left(\tilde{N}_{1}+1\right)}=\left(\tilde{N}_{1} v_{0}+\max \left\{\tilde{N}_{1}, \tilde{N}_{2}\right\}\right) T_{p}$. 
- The first projection value along the paths in (p2) reaches the $\mathrm{FC}$ at most at $t_{f}^{N_{1}+2}=\left[\left(\tilde{N}_{1}+1\right) \nu_{0}+\max \left\{\tilde{N}_{1}, \tilde{N}_{2}\right\}\right] T_{p}$.

- The last projection value along the paths in (p2) reaches the FC at most at

$$
t_{f}^{\tilde{N}_{1}+\tilde{N}_{2}+1}=\left[\left(\tilde{N}_{1}+\tilde{N}_{2}+1\right) v_{0}+\tilde{N}_{1}\right] T_{p} .
$$

Overall, the $\pi / 4$-oriented diagonal projections in a quadrant are performed after $\left[\left(\tilde{N}_{1}+\tilde{N}_{2}+1\right) \nu_{0}\right.$ $\left.+\max \left\{\tilde{N}_{1}, \tilde{N}_{2}\right\}\right]$ time slots. Again, if the quadrants are processed in a serial fashion, the overall number of time slots needed to evaluate the diagonal projections sums up to

$$
\begin{aligned}
N_{\mathrm{TS}}^{(\pi / 4)} & =2\left[\left(N_{1}+N_{2}\right) v_{0}+\left(\max \left\{N_{1}, N_{2}\right\}-1\right)\right] \\
& =2 \sqrt{N}\left[\left(\alpha_{1}+\alpha_{2}\right) \nu_{0}+\max \left\{\alpha_{1}, \alpha_{2}\right\}\right]-2 .
\end{aligned}
$$

Figure 15 reports, for the sake of clarity, a time diagram summarizing the timing of projection evaluation when performing diagonal projections within a quadrant of the network.

Then if we consider a Radon-like scheme comprising $P=3$ projections along the directions $\vartheta=0, \vartheta=$ $\pi / 2, \vartheta=\pi / 4$, the overall number of needed transmissions sums up to (cfr. (25), (27), and (29))

$$
\begin{aligned}
& N_{\mathrm{TX}}^{(0, \pi / 2, \pi / 4)} \approx \gamma_{3} N \\
& \gamma_{3}=\left(2 \alpha_{1} \alpha_{2}+\frac{\alpha_{1}^{2}+\alpha_{2}^{2}}{2}\right)+\left(\alpha_{1}+\alpha_{2}\right) \max \left\{\alpha_{1}, \alpha_{2}\right\}
\end{aligned}
$$

while the number of needed time slots is (cfr. (26), (28), and (30))

$$
\begin{aligned}
& N_{\mathrm{TS}}^{(0, \pi / 2, \pi / 4)} \approx \delta_{3} \sqrt{N} \\
& \delta_{3}=\left(\alpha_{1}+\alpha_{2}\right)\left(4+4 \nu_{0}\right)+\max \left\{\alpha_{1}, \alpha_{2}\right\} .
\end{aligned}
$$

To recap, the Radon-like CS data gathering procedure lets the fusion center collect all the needed measurements

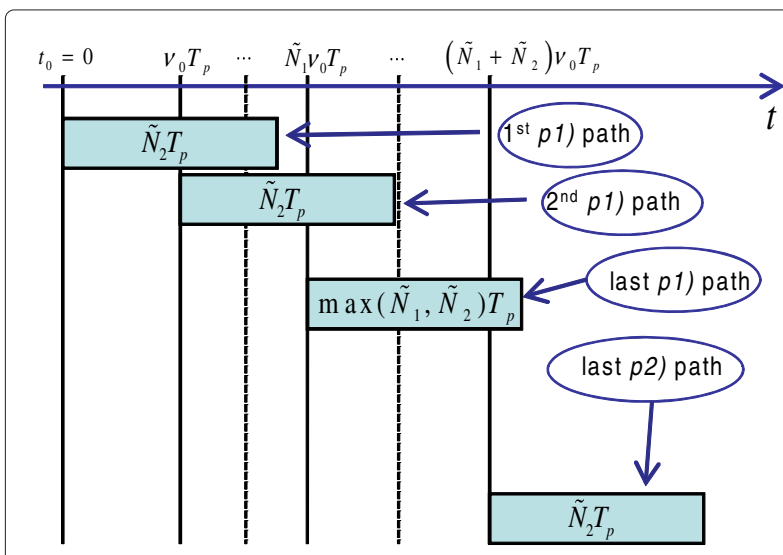

Figure 15 Data gathering algorithm: timing of the sensors' transmissions for diagonal projections evaluation. in a highly parallelized fashion. Far from being optimal, the data gathering scheme introduced herein allows a significant reduction in both the occupied bandwidth and the consumed energy w.r.t. state-of-the-art data gathering scheme such as the RS introduced in [10]. Further developments of globally optimized Radon-like CS data gathering algorithms are still under investigation.

\section{Competing interests}

The authors declare that they have no competing interests.

Received: 17 January 2013 Accepted: 18 July 2013

Published: 12 August 2013

\section{References}

1. S Colonnese, T Melodia, F Cuomo, in Proceedings of Global Communications Conference (GLOBECOM) 2012. Leveraging multiview video coding in clustered multimedia sensor networks (Anaheim, 3-7 December 2012)

2. DS Ghataoura, JE Mitchell, GE Matich, Networking and application interface technology for wireless sensor network surveillance and monitoring. IEEE Commun. Mag. 49(10), 90-97 (2011)

3. JP Sheu, WK Hu, JC Lin, Distributed localization scheme for mobile sensor networks. IEEE Trans. Mobile Comput. 9(4), 516-526 (2010)

4. NAA Aziz, KA Aziz, in Proceedings of the International Conference on Advanced Communication Technology (ICACT) 2011. Managing disaster with wireless sensor networks (Seoul, 13-16 February 2011)

5. M Carli, S Panzieri, F Pascucci, A joint routing and localization algorithm for emergency scenario. Ad Hoc Networks (2012). doi:10.1016/j.adhoc.2012.09.001

6. F Ren, J Zhang, T He, C Lin, SK Das, EBRP: energy-balanced routing protocol for data gathering in wireless sensor networks. IEEE Trans. Parallel Distributed Syst. 22(12), 2108-2125 (2011)

7. YEM Hamouda, C Phillips, Adaptive sampling for energy-efficient collaborative multi-target tracking in wireless sensor networks. IET Wireless Sensor Syst. 1(1), 15-25 (2011)

8. RG Baraniuk, E Candés, M Elad, Yi Ma, Applications of sparse representation and compressive sensing. Proc. IEEE. 98(6), 906-909 (2010)

9. G Quer, R Masiero, D Munaretto, M Rossi, J Wider, M Zorzi, in Proceedings of the Information Theory and Applications Workshop 2009. On the interplay between routing and signal representation for compressive sensing in wireless sensor networks (San Diego, 8-13 February 2009)

10. F Fazel, M Fazel, M Stojanovic, Random access compressed sensing for energy-efficient underwater sensor networks. IEEE J. Select. Areas Commun. 29(8), 1660-1670 (2011)

11. EJ Candés, MB Wakin, An introduction to compressive sampling IEEE Signal Proc. Mag. 25(2), 21-30 (2008)

12. RG Baraniuk, M Davenport, R DeVore, M Wakin, A simple proof of the restricted isometry property for random matrices. Constr. Approximation. 28(3), 253-263 (2008)

13. J Tropp, AC Gilbert, Signal recovery form partial information via orthogonal matching pursuit. IEEE Trans. Inf. Theory. 53, 4655-4666 (2007)

14. D Needell, J Tropp, CoSaMP: Iterative signal recovery form incomplete and inaccurate samples. Appl. Comput. Harmonic Anal. 26, 301-321 (2008)

15. W Bajwa, J Haput, A Sayeed, R Nowak, in Proceedings of the 5th International Conference on Information Processing Sensor Networks (ISPNO6). Compressive wireless sensing (ACM, New York, 2006), pp. 134-142

16. G Shen, SY Lee, S Lee, S Pattem, A Tu, B Krishnamachari, A Ortega, M Cheng, S Dolinar, A Kiely, M Klimesh, H Xie, in Proceedings Electronics System-Integration Technology Conference (ESTC-2008). Novel distributed wavelet transforms and routing algorithms for efficient data gathering in sensor webs (London, 1-4 September 2008)

17. C Caione, D Brunelli, L Benini, Distributed compressive sampling for lifetime optimization in dense wireless sensor networks. IEEE Trans. Industrial Informatics. 8(1), 30-40 (2012)

18. C Luo, F Wu, J Sun, CW Chen, Efficient measurement generation and pervasive sparsity for compressive data gathering. IEEE Trans. Wireless Commun. 9(12), 3728-3738 (2010) 
19. SLee, A Ortega, in APSIPA Annual Summit and Conference (APSIPA ASC 2010). Joint optimization of transport cost and reconstruction for spatially-localized compressed sensing in multi-hop sensor networks (Singapore, 14-17 December 2010)

20. S Colonnese, P Campisi, G Panci, G Scarano, Blind image deblurring driven by nonlinear processing in the edge domain. EURASIP J. Appl. Signal Process. 16, 2462-2475 (2004)

21. F Matus, J Flusser, Image representation via a finite Radon transform. IEEE Trans. on Pattern Anal. Mach. Intell. 15(10), 996-1006 (1993)

22. S Colonnese, R Cusani, S Rinauro, G Scarano, in 5th International Symposium on Communications Control and Signal Processing. Computationally-efficient compressive sampling of pulse stream images using Radon-like measurements (Rome, 2-4 May 2012)

23. HL Yap, A Eftekhari, MB Wakin, CJ Rozell, in Proceedings of the Conference on Information Sciences and Systems (CISS). The restricted isometry property for block diagonal matrices (Baltimore, 23-25 March 2011)

24. J Haupt, WU Bajwa, M Rabbat, R Nowak, Compressed sensing of networked data. IEEE Signal Proc. Mag. 25(2), 92-101 (2008)

25. C Hegde, RG Baraniuk, Sampling and recovery of pulse streams. IEEE Trans. Sig. Proc. 59(4), 1505-1517 (2011)

26. NASA: http://ourocean.jpl.nasa.gov./ Accessed March 2013

27. SS Kanhere, in 12th IEEE International Conference on Mobile Data Management (MDM 2011). Participatory sensing: crowdsourcing data from mobile smartphones in urban spaces (Lulea, 6-9 June 2011)

28. M Kendall, A Stuart, JK Ord, Kendall's Advanced Theory of Statistics, Volume 1: Distribution Theory, 6th edn. (Oxford University Press, Oxford, 1994)

29. JB Proakis, Digital Communications, 5th edn. (McGraw-Hill, New York, 2007)

30. S Colonnese, S Rinauro, R Cusani, G Scarano, The restricted isometry property of the Radon-like CS matrix. Proc. of IEEE Int. Work. on Multimedia Signal Processing, 2013 in press

doi:10.1186/1687-6180-2013-136

Cite this article as: Colonnese et al:: Efficient compressive sampling of spatially sparse fields in wireless sensor networks. EURASIP Journal on Advances in Signal Processing 2013 2013:136.

\section{Submit your manuscript to a SpringerOpen ${ }^{\circ}$ journal and benefit from:}

- Convenient online submission

Rigorous peer review

- Immediate publication on acceptance

- Open access: articles freely available online

- High visibility within the field

- Retaining the copyright to your article

Submit your next manuscript at $\boldsymbol{\nabla}$ springeropen.com 